\title{
ArcheoSciences
}

Revue d'archéométrie

\section{Paysages, productions et collectes végétales en Limousin (Massif central occidental) de la Tène finale à la fin du Moyen Âge (100 BC-1 400AD)}

Landscapes, Plant Production and Gathering in Limousin (Western Massif central) between the Later La Tène Period and the Late Middle Ages (100 BC-1400 $A D)$

Anne Bouchette, Manfred Rösch et Guy Jalut

\section{(2) OpenEdition}

\section{Édition électronique}

URL : https://journals.openedition.org/archeosciences/2932

DOI : $10.4000 /$ archeosciences. 2932

ISBN : 978-2-7535-1849-0

ISSN : $2104-3728$

\section{Éditeur}

Presses universitaires de Rennes

Édition imprimée

Date de publication : 30 avril 2011

Pagination : 65-92

ISBN : 978-2-7535-1847-6

ISSN : $1960-1360$

\section{Référence électronique}

Anne Bouchette, Manfred Rösch et Guy Jalut, « Paysages, productions et collectes végétales en Limousin (Massif central occidental) de la Tène finale à la fin du Moyen Âge (100 BC-1 400AD) », ArcheoSciences [En ligne], 35 | 2011, mis en ligne le 30 avril 2013, consulté le 28 février 2022. URL : http://journals.openedition.org/archeosciences/2932; DOI : https://doi.org/10.4000/archeosciences. 2932 


\title{
Paysages, productions et collectes végétales en Limousin (Massif central occidental) de la Tène finale à la fin du Moyen Âge (100 BC-1 400AD)
}

\author{
Landscapes, Plant Production and Gathering in Limousin (Western Massif central) \\ between the Later La Tène Period and the Late Middle Ages (100 BC-1400 AD)
}

\author{
Anne Bouchette*, Manfred Rösch**, Guy Jalut***
}

\begin{abstract}
Résumé : Entre 1996 et 2004, plusieurs sites archéologiques du Limousin ont fait l'objet d'une analyse carpologique. Bien que le nombre de sites et de structures analysés soit modeste, près de 219000 restes carbonisés, imbibés ou minéralisés et environ 300 taxons de plantes cultivées et sauvages ont été déterminés permettant l'élaboration d'une première synthèse pour une période allant de la Tène finale au bas Moyen Âge. Ce travail intègre également la publication de plusieurs études carpologiques de nécropoles gallo-romaines ainsi que les acquis régionaux des recherches anthracologiques, palynologiques et sédimentologiques. La synthèse sur les plantes cultivées et utilitaires met en évidence une modification des productions céréalières entre la fin de l'époque gallo-romaine et le haut Moyen Âge, caractérisée par la montée en puissance du seigle (Secale cereale). Elle rend compte aussi d'un développement de l'arboriculture à partir de l'époque gallo-romaine, puis au haut Moyen Âge, notamment en contexte urbain. Des importations ont également été mises en évidence, comme le poivre (Piper nigrum) au haut-Empire.
\end{abstract}

\begin{abstract}
Between 1996 and 2004 palaeobotanical studies were performed in archaeological sites of Limousin. Despite the limited number of the sites and structures studied, the richness in plant macroremains and taxa strenghthened by the use of additional palaeobotanical studies as well as anthracological, palynological, sedimentological and palaeoclimatic data allows to propose a first synthesis concerning the agricultural production between the end of La Tene and the low Middle Ages. More than 219.000 remains (carbonized, waterlogged and mineralized) and circa 300 wild and cultivated plants taxa were determined. This work emphasizes a change in the cereal production between the end of the Gallo-Roman period and the High Middle Age characterized by the increase in rye (Secale cereale) cultivation. It also shows the development of arboriculture during the Gallo-Roman period and the high Middle Ages, especially in urban area. The import of an exotic spice : pepper (Piper nigrum), is demonstrated during the High Empire.
\end{abstract}

Mots clé : Massif central, Limousin, carpologie, $2^{\mathrm{e}}$ âge du Fer, époque gallo-romaine, Moyen Âge.

Keywords : Massif central, Limousin, palaeoethnobotany, $2^{\text {nd }}$ Iron Age, Gallo-Roman period, Middle Ages.

* INRAP, 561, rue Étienne-Lenoir, Km Delta, F-30900 Nîmes et UMR 5140 - Archéologie des Sociétés Méditerranénnes. (anne.bouchette@inrap.fr)

** Landesamt für Denkmalpflege im Regierungspräsidium Stuttgart, Fischersteig 9, D-78 343 Hemmenhofen, Germany

*** Laboratoire d'Écologie Fonctionnelle et Environnement (ECOLAB), UMR 5245 CNRS-UPS-CNPT, Université Paul-Sabatier, Campus UPS, Bât. 4R1, 118 route de Narbonne, 31062 Toulouse cedex 9. 


\section{INTRODUCTION}

Jusqu'à ces dernières années, le Limousin a été une région pour laquelle le nombre d'études carpologiques est resté limité. Dans une synthèse sur les données néolithiques et protohistoriques de la France réalisée par M. Hopf (1991), le Limousin était la seule région à ne pas figurer dans cet inventaire. Des études inédites avaient toutefois été réalisées entre 1984 et 1988 , en particulier sur des sites du Néolithique et de l'âge du Bronze (dolmens de La Lue, Berneuil [Haute-Vienne]) et du Petit-Pied, SaintCernin-de-Larche (Corrèze) et un site médiéval (Chadalais, Maisonnais-sur-Tardoire [Haute-Vienne]) ${ }^{1}$. Mais, c'est seulement au début des années 1990 que les premières études carpologiques de sites gallo-romains (Marinval, 1991, 1993, 2001, Roger, 1995) ont fait l'objet de publications. D'autres études ont depuis été publiées comme celles de la nécropole gallo-romaine des Châtaigneraies à Saint-Germain-les-Vergnes (Corrèze) (Cabanis, in Toledo i Mur et al., 2004) et celles du Néolithique ancien et récentfinal ci-dessus citées (Tardiveau et al., 1990; Ruas, 2001; Diot, 2002).

Entre 1996 et 2001, 9 sites couvrant une période allant de la Tène finale au bas Moyen Âge ont été étudiés, permettant la détermination d'environ 212000 restes végétaux et 300 taxons $^{2}$ de plantes cultivées et sauvages (Bouchette, 2004). Dans le cadre de cette présentation, seules les données concernant les plantes cultivées et utilitaires des périodes protohistorique, gallo-romaine et médiévale ont été retenues (tableau 1). Ce choix s'est imposé pour deux raisons : l'une technique, liée à la longueur des tableaux contenant la totalité du corpus carpologique; l'autre scientifique, due au fait qu'aucune nouvelle donnée ne concerne les périodes néolithique et de l'âge du Bronze.

Le Limousin bénéfice de nombreuses études palynologiques, anthracologiques et sédimentologiques qui permettent de rendre compte des variations climatiques ou anthropo-climatiques pour les périodes protohistoriques et historiques. Les études montrent montrent une certaine hétérogénéité par rapport aux données carpologiques en raison de la localisation géographique de ces dernières. Ainsi, la plupart des analyses palynologiques sont localisées en Montagne limousine et proviennent de tourbières alors que les données carpologiques et anthracologiques résultent de sites de vallées et de bas-plateaux (Dubois et al., 1942; Dubois et Dubois, 1944; Lemée, 1943, 1949, 1952, 1980 ;

1. Réalisées par M.-P. Ruas.

2. Ont été retenues comme taxons les déterminations réalisées au rang d'espèce et de genre. Les déterminations incertaines (cf.) n’ont été prises en considération que lorsque le taxon n'est pas attesté sous une autre forme.

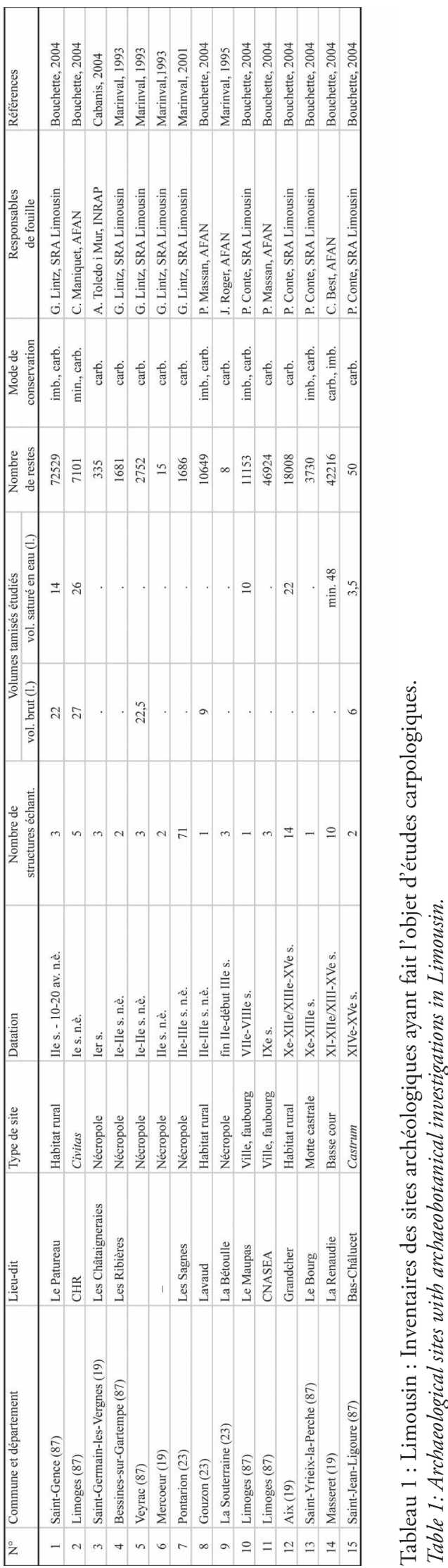

ArCheoSCIEnCes, revue d'archéométrie, 35, 2011, p. 65-92 
Denèfle et al., 1980; Guenet, 1992, 1993; Miras, 2001, 2004). Plusieurs analyses palynologiques, réalisées dans le cadre du Programme Collectif de Recherche sur « l'archéologie agraire en Limousin ", concernent néanmoins des fonds de vallons tourbeux des bas-plateaux occidentaux (Allée et Diot, 1997; Allée et al., 1996, 1997). Les données anthracologiques publiées (Fredon, 1985, 1995) ont rarement fait l'objet d'analyses carpologiques ou palynologiques complémentaires.

\section{Le Limousin}

\section{Le cadre géographique et géologique}

Le Limousin forme la partie nord-occidentale du Massif central. Composé de trois départements, la Corrèze (19), la Creuse (23) et la Haute-Vienne (87), sa superficie est d'environ $17000 \mathrm{~km}^{2}$ (fig. 1). Son relief est caractérisé par quatre grandes entités :

- les hauts sommets, localement appelés Montagne limousine, dont l'altitude varie de 600 à $978 \mathrm{~m}$,

- les plateaux, situés entre $200 \mathrm{~m}$ et $600 \mathrm{~m}$ d'altitude,

- les vallées, nombreuses du fait de l'importance du réseau hydrographique,

- deux petits bassins sédimentaires dont celui de Gouzon au nord-est et celui de Brive au sud.

Le Limousin est principalement composé d'un socle cristallin arénisé. Dans l'ensemble, les sols y sont légers, acides, froids et humides. Les sols podzoliques et hydromorphes dominent en altitude, notamment en Montagne limousine (Bernard-Allée et al., 1994; Brugel et al., 2001). C'est sur ce relief que se localisent les tourbières les plus vastes et les plus anciennes. Dans les vallées, les sols alluviaux l'emportent. Les affleurements calcaires se limitent pour l'essentiel au Causse de Brive.

\section{Climat et végétation actuels}

Le climat régional est majoritairement de type tempéré océanique. Dans le nord-est de la Creuse, il prend un caractère plus continental mais devient plus méridional dans le bassin de Brive et sur le Causse corrézien. Du fait du relief, la pluviométrie est fortement contrastée. Avec des précipitations annuelles supérieures à $1500 \mathrm{~mm}$, la Montagne limousine fait partie des zones les plus fortement arrosées. Les bas-plateaux et les deux bassins connaissent des précipitations inférieures à $900 \mathrm{~mm} / \mathrm{an}$ (Walter et al., 1960).

Trois grandes régions paysagères ont été identifiées (Périgord, 1994) :
- dans le nord et nord-ouest du Limousin, un type bocager à habitat dispersé,

- dans l'ouest et le sud-ouest, domaine de l'élevage bovin, des pâturages et un habitat regroupé,

- dans l'est et le sud-est, un milieu façonné par une activité agro-sylvo-pastorale.

La végétation naturelle potentielle fait référence aux séries du chêne pédonculé (Quercus robur), du chêne pubescent (Q. pubescens), du chêne sessile (Q. petraea), du hêtre (Fagus sylvatica) et de l'aulne (Alnus glutinosa) (Lavergne, 1969). Elles s'articulent les unes aux autres selon l'altitude, le sol, le relief et l'exposition. Si la série du hêtre prédomine sur la Montagne limousine, les massifs des plateaux sont davantage caractérisés par la série du chêne pédonculé, les bas plateaux par celles des chênes sessile et pédonculé. Celle du chêne pubescent n'est localisée que dans le bassin de Brive et le Causse de Martel (Lavergne, 1963). Dans les vallées, la végétation potentielle des ripisylves est formée par la série de l'aulne.

\section{Matériel et MÉTHOdes}

\section{Le corpus des sites}

Les données carpologiques prises en compte font référence à 15 sites, soit 18 occupations (tableau 1 et fig. 1). Bien que relevant des périodes considérées, les deux sites médiévaux de Chadalais à Maisonnais-sur-Tardoire (Haute-Vienne) et Esplaux à Sarran (Corrèze) n'ont pas été retenus dans la mesure où, jusqu'à présent, les données carpologiques n'ont fait l'objet d'aucune publication détaillée (Ruas, 1992a; Colombain et Lombard, 1991). Seules les plantes cultivées sont diponibles sous forme de présence/absence pour le premier et de mention pour le second. Il en va de même pour les sites de Saint-Christophe et de Louroux (Creuse) dont les déterminations n'émanent pas de spécialistes.

Les sites pris en considération se répartissent de façon inégale. Quatre d'entre eux sont localisés en Corrèze, trois en Creuse et huit en Haute-Vienne (fig. 1). Géographiquement, leur distribution fait référence pour l'essentiel au Limousin occidental, c'est-à-dire aux bas-plateaux et aux vallées. Ceux implantés en moyenne montagne font exception et aucun ne rend compte de la haute Montagne. Aussi, en termes de végétation naturelle potentielle, les séries concernées sont principalement celles des différents chênes et de l'aulne.

Du point de vue distribution chronologique, les occupations retenues se répartissent de manière inégale : une seule est datée de la Tène finale, neuf font référence à l'époque gallo-romaine et huit au Moyen Âge. Les types d'occupa- 


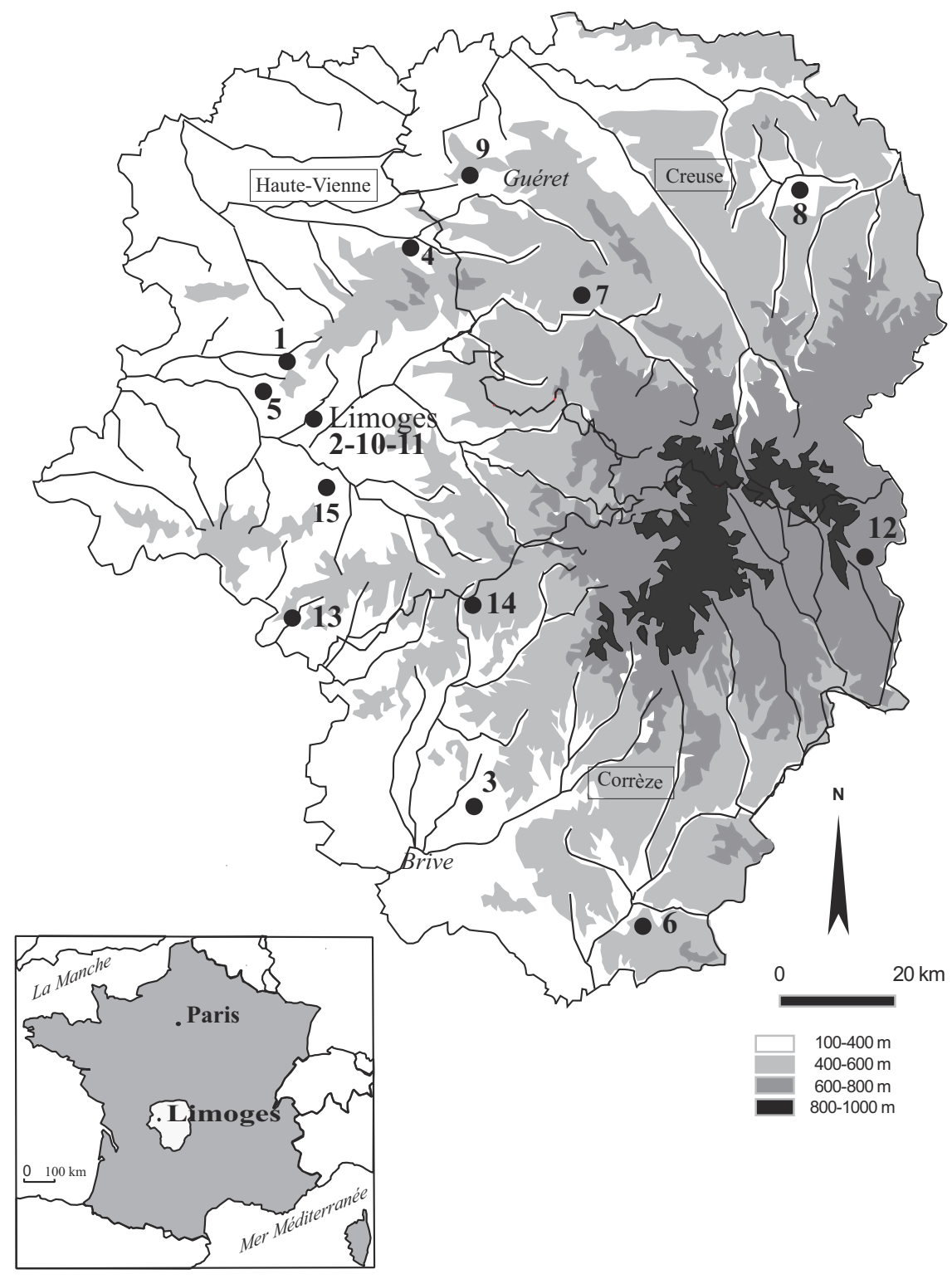

Figure 1 : Limousin : localisation des sites.

Figure 1: Limousin : location of the sites.

tion diffèrent également. Ainsi, le site gaulois et antique du Patureau à Saint-Gence est interprété comme un centre proto-urbain (Lintz, 2000). Les autres données carpologiques gallo-romaines proviennent pour une large part de nécropoles à incinérations. Deux habitats, l'un urbain, l'autre rural, complètent le spectre. Pour le Moyen Âge, les sites retenus sont constitués par différentes occupations urbaines, castrales et rurales.

\section{Les contextes archéologiques et les modes de conservation}

Les contextes archéologiques analysés sont variés. Les sites retenus totalisent 124 structures, soit 8 structures en moyenne. Ces chiffres masquent une forte hétérogénéité dans la mesure où la nécropole de Sagnes à Pontarion a fourni à elle seule 71 contextes. Excepté ce site, le nombre de structures analysées varie de 1 à 14 . Certaines d'entre elles ont donné lieu à des prélèvements très riches en carpo-restes (tableau 2).

Plusieurs structures d'ambiance humide ont fait l'objet de prélèvements : trois puits à Saint-Gence-le Patureau, un à Gouzon-Lavaud et un à Limoges-le Maupas, un fossé à Saint-Yrieix-la-Perche-le Bourg et un souterrain à Masseret-la Renaudie. Les comblements se sont révélés plus ou moins organiques selon les structures. Les autres contextes sont de milieu sec et concernent la majorité des sites. Ils sont constitués par des incinérations, des fosses, des 


\begin{tabular}{|c|c|c|c|c|c|c|}
\hline \multirow[t]{2}{*}{ Période et commune } & \multirow[t]{2}{*}{ Site } & \multicolumn{3}{|c|}{ Fossilisation - $\% /$ nbre de restes } & \multicolumn{2}{|r|}{ Structures } \\
\hline & & Carbonisation & Imbibition & Minéralisation & Nombre & Types \\
\hline \multicolumn{7}{|l|}{ Age du Fer } \\
\hline Saint-Gence & Le Patureau & 1,26 & 98,7 & . & 2 & puits \\
\hline \multicolumn{7}{|l|}{ Gallo-Romain } \\
\hline Saint-Gence & Le Patureau & 0,01 & 99,9 & . & 1 & puits \\
\hline Limoges & $\mathrm{CHR}$ & 18,6 & . & 81,4 & 5 & fosse, puits/latrines? \\
\hline $\begin{array}{l}\text { St-Germain-les- } \\
\text { Vergnes }\end{array}$ & Les Châtaigneraies & 100 & . & . & 3 & sépultures \\
\hline $\begin{array}{l}\text { Bessines-sur- } \\
\text { Gartempe }\end{array}$ & Les Ribières & 100 & . & . & 2 & sépultures \\
\hline Veyrac & Veyrac & 100 & . & . & 3 & sépultures \\
\hline Mercoeur & Mercoeur & 100 & . & . & 2 & sépultures \\
\hline Gouzon & Lavaud & 1,3 & 98,6 & . & 1 & puits \\
\hline Pontarion & Les Sagnes & 100 & . & . & 71 & sépultures \\
\hline La Souterraine & La Bétoulle & 100 & . & . & 3 & sépultures \\
\hline \multicolumn{7}{|l|}{ Moyen Age } \\
\hline Limoges & Le Maupas & 2,16 & 97,8 & . & 1 & puits \\
\hline Limoges & Cnasea & 100 & . & . & 3 & fosses \\
\hline St Yrieix & Le Bourg & 0,03 & 99,7 & . & 1 & fossé \\
\hline Aix & Grandcher & 100 & . & . & 14 & $\begin{array}{l}\text { fosses, bâtiments, } \\
\text { four, foyer }\end{array}$ \\
\hline Masseret & La Renaudie (X-XII) & 100 & . & . & 8 & fosses, fossé \\
\hline Masseret & La Renaudie (XII-XV) & 7,9 & 92,1 & . & 2 & fosse, souterrain \\
\hline Saint-Jean-Ligoure & Bas-Chalucet & 100 & . & . & 2 & niveaux d'occupation \\
\hline TOTAL & & & & & 124 & \\
\hline
\end{tabular}

trous de poteau, des niveaux et des remblais d'occupation, des fours et des foyers. Quatre structures seulement, provenant toutes du site du CHR de Limoges, ont livré majoritairement du matériel minéralisé (Maniquet [dir.], 1999) (tableau 2).

La composition des assemblages carpologiques de ces contextes divergent dans la mesure où les modes de conservation influent largement sur la préservation des restes végétaux. Ainsi, selon Green (1979), en milieu urbain, les structures d'ambiance humide sont plus favorables à la conservation des fruitiers que des céréales et des légumineuses. Cette observation est valable pour le Limousin avec le puits du site urbain de Limoges-le Maupas, et à un degré moindre, pour le fossé de la motte castrale de Saint-Yrieixla-Perche-le Bourg. Par contre, cette constatation s'avère moins pertinente en ce qui concerne les occupations non urbaines. Ainsi, le souterrain du site castral de Masseret-la Renaudie, pour lequel le matériel imbibé prédomine, est composé principalement de résidus de céréales et d'oléagineuses. À Saint-Gence-le Patureau, habitat rural ou protourbain gaulois et gallo-romain, les trois puits analysés ont livré un abondant matériel imbibé, caractérisé par un spectre de plantes cultivées et utilitaires où les résidus de céréales abondent (fig. 2). Conformément aux observations de Green (1979), les contextes archéologiques de milieu sec ont livré, pour l'essentiel, des céréales et/ou des légumineuses carbonisées. Cette représentation est surtout valable pour les différentes occupations médiévales, quelle que soit leur nature. Elle l'est moins pour les sites gallo-romains, car la plupart d'entre eux sont constitués de nécropoles à incinérations. Ce type de dépôts favorise la mise en évidence d'un spectre de fruits plus ou moins diversifié (Marinval, 1993; Bouby et Marinval, 2004), même si en Limousin, les corpus sont constitués majoritairement de céréales et de légumineuses. Troisième mode de conservation présent sur les sites retenus, la minéralisation n'est attestée en Limousin que dans quatre structures gallo-romaines à Limoges-CHR.

\section{Le matériel carpologique}

Les prélèvements que nous avons étudiés ont été tamisés à l'eau sur une colonne de tamis à mailles de $2 \mathrm{~mm}, 1 \mathrm{~mm}$ et $0,5 \mathrm{~mm}$ pour les prélèvements issus de milieux secs. Pour ceux d'ambiance humide, un tamis à maille de $0,2 \mathrm{~mm}$ a été ajouté. Les quantités prélevées varient selon les sites, les contextes, les couches archéologiques, la nature des comblements, etc. Les refus de tamis de $2 \mathrm{~mm}$ ont été triés dans leur intégralité. Les autres mailles ont parfois dû faire l'objet d'un sous-échantillonnage du fait de leur volume, de la densité des restes conservés ou du temps dont nous disposions pour le travail. Lorsqu'un tri partiel des fractions a dû être opéré, le nombre de restes a été calculé au prorata des pourcentages 


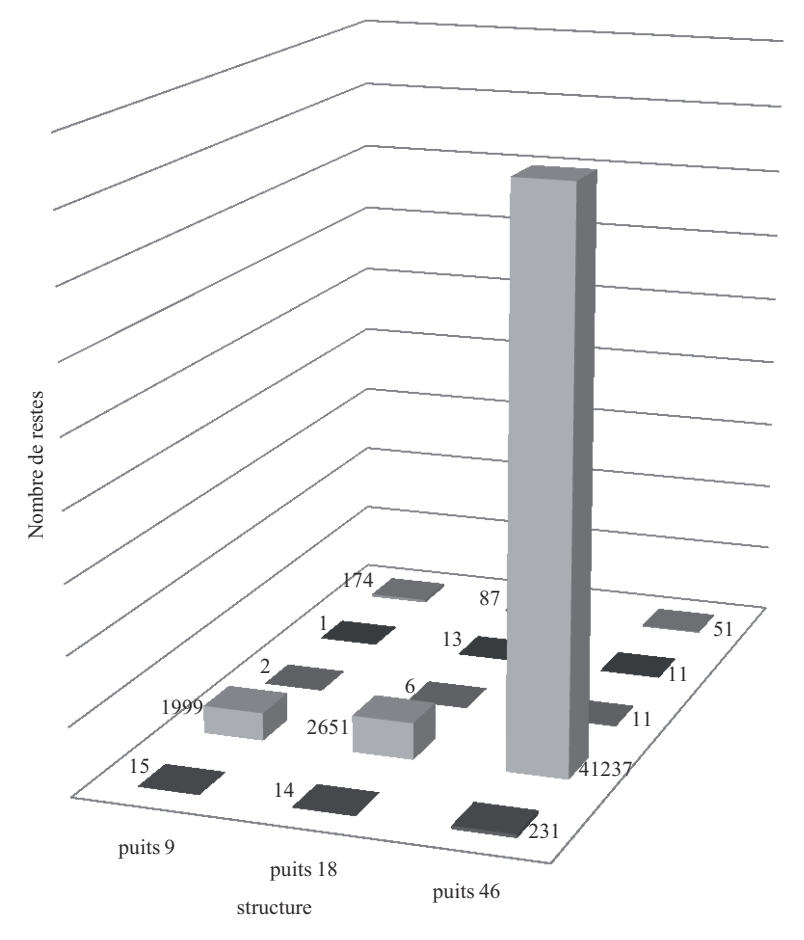

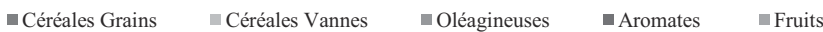

Figure 2 : Saint-Gence (87), le Patureau : nombre de restes par puits. Figure 2: Saint-Gence (87), le Patureau: number of macroremains per well.

effectués afin d'obtenir une estimation globale du contenu de l'échantillon. Pour les sites étudiés, le nombre de déterminations s'élève à 212360 restes de plantes cultivées et sauvages (tableau 3 et 4). Il s'agit d'un nombre minimum d'individus, ceux-ci pouvant être entiers ou composés de fragments. Dans ce dernier cas, le mode de calcul a été établi de la manière suivante : deux moitiés de semences ou cinq fragments équivalant à un reste. Un mode de calcul similaire a été retenu pour les sites que nous n'avons pas étudiés et pour lesquels le nombre de fragments a été divisé par 5 lorsqu'il était exprimé. Ainsi, le total des déterminations du corpus des sites avoisine les 219000 restes de plantes cultivées et sauvages pour environ 300 taxons. 241 d'entre eux ont été déterminés au rang d'espèce. Réduit aux plantes cultivées et utilitaires retenues, le nombre d'identifications s'établit à 154035 éléments pour 70 taxons dont 58 espèces.

\section{Travail de laboratoire}

Les échantillons étudiés ont été tamisés soit au Laboratoire d'Ecologie Terrestre (université Paul-Sabatier, Toulouse) soit à l'Institut d'Archéobotanique du Landesdenkmalamt
Baden-Wurttemberg de Hemmenhofen-Gaienhofen (Allemagne). D'autres l'ont été par les équipes de fouille. Les volumes des prélèvements ne sont connus qu'en partie, ce qui n'a pas permis de calculer la densité en restes pour l'ensemble des sites. Ils sont compris, en données brutes, entre 6 à 27 litres et en données saturées en eau entre 3,5 à 48 litres (tableau 1). Ces valeurs relativement faibles s'expliquent par le fait que les prélèvements ont été pour la plupart réalisés sur des sédiments contenant des carpo-restes visibles à l'œil nu.

Les restes végétaux ont ensuite été triés et déterminés à l'aide d'un stéréomicroscope à des grossissements x10 ou x60. Les déterminations ont été réalisées à partir d'atlas de semences, d'articles spécialisés et de collections de référence de fruits et de graines actuels.

\section{RÉsultats}

\section{Lâge du Fer}

\section{L'environnement végétal d'après les données palynologiques}

Au début de l'âge du Fer, le paysage végétal du Limousin est encore largement dominé par la forêt. Selon l'altitude, il est constitué de chênaies (Quercetum) ou de chênaieshêtraies (Querceto-fagetum). En Montagne limousine, les hêtraies dominent et sont plus ou moins parsemées de sapins (Abies). Comme cela a été montré dans les Pyrénées (Kenla et Jalut, 1979; Jalut, 1984) où le sapin peut se développer à basse et moyenne altitude, la dominance locale du hêtre par rapport au sapin pourrait être l'une des conséquences de l'action de l'homme. Les fonds de vallées et les berges des tourbières sont colonisés par les aulnaies (Alnetum) (Lemée, 1943, 1949, 1980; Denèfle et al., 1980; Guenet, 1992, 1993; Allée et al., 1996, 1997 ; Miras, 2001, 2004). Des grains de pollen de graminées (Poaceae) et de rudérales témoignent de l'anthropisation du paysage. Ceux des Ericacées traduisent à la fois une présence locale sur les tourbières ainsi que la probable existence de landes dont le développement et l'emprise restent difficile à évaluer.

\section{Productions et collectes végétales}

Au second âge du Fer, l'agriculture en Limousin est caractérisée par des attestations polliniques de céréales et des données carpologiques. Ces dernières, datées de la Tène finale, proviennent de Saint-Gence-le Patureau. Cette occupation, localisée au nord-ouest de Limoges, correspond à un village bien structuré d'époque gauloise et augustéenne (Lintz, 


\begin{tabular}{|c|c|c|c|c|c|c|c|c|c|c|c|c|}
\hline $\mathrm{N}^{\circ}$ sites & & La Tène & & & & & Ilo-rom & & & & & \\
\hline Chror & & 1 & 1 & 2 & 3 & 4 & 5 & 6 & 7 & 8 & 9 & \\
\hline & & LTf & & & $\mathrm{Ht}$ & & & & & $\mathrm{Ht} / \mathrm{BsF}$ & & \\
\hline Structures & & $\mathrm{Hr}$ & $\mathrm{Hr}$ & Civ & $\mathrm{N}$ & $\mathrm{N}$ & $\mathrm{N}$ & $\mathrm{N}$ & $\mathrm{N}$ & $\mathrm{Hr}$ & $\mathrm{N}$ & \\
\hline Conservation & & $\mathrm{p}$ & $\mathrm{p}$ & $f, 1$ & inc. & inc & inc & inc & inc & $\mathrm{p}$ & inc. & \\
\hline Taxons & Etat & $\mathrm{i}, \mathrm{c}$ & $\mathrm{i}, \mathrm{c}$ & $\mathrm{c}, \mathrm{m}$ & c & c & c & $\mathrm{c}$ & c & $\mathrm{i}, \mathrm{c}$ & c & Nom français \\
\hline Céréales & & & & & & & & & & & & \\
\hline Grains & & & & & & & & & & & & \\
\hline Avena & carb. & 3 & 3 & 8 & 1 & . & 26 & . & 26 & . & . & Avoine \\
\hline Avena sativa, grain vêtu & carb. & . & . & . & . & . & 7 & . & . & . & . & Avoine cultivée \\
\hline Hordeum vulgare & carb. & . & . & 1223 & $14,5 \mathrm{ml}$ & 373 & 1502 & . & 115 & 2 & 1 & Orge vêtue \\
\hline Hordeum vulgare var. nudum & carb. & . & . & . & 31 & . & . & . & . & . & . & Orge nue \\
\hline Hordeum & imb. & . & 6 & . & . & . & . & . & . & . & . & Orge \\
\hline Hordeum & carb. & . & . & . & . & . & . & . & . & . & 1 & Orge \\
\hline Triticum aestivum/durum & carb. & 1 & . & 8 & 8 & . & . & . & 146 & 1 & . & Blé tendre/dur \\
\hline Triticum dicoccon & imb. & . & 1 & . & . & . & . & . & . & . & . & Amidonnier \\
\hline Triticum dicoccon & carb. & . & 3 & . & . & 826 & . & . & 698 & 1 &. & Amidonnier \\
\hline Triticum monococcum & carb. & . & . & . & . & . & . & . & 1 & . & . & Engrain \\
\hline Triticum dicoccon/monococcum & carb. & . & . & . & 1 & . & . & . & . & . & . & Amidonnier/Engrain \\
\hline Triticum spelta & carb. & 2 & 6 & . & . & . & 505 & . & . & 1 &. & Epeautre \\
\hline Triticum & imb. & 10 & 181 & . & . & . & . & . & . & . & . & Blé \\
\hline Triticum & carb. & 2 & 2 & 1 & 15 & . & . & . & 22 & . & . & Blé \\
\hline Panicum miliaceum & carb. & 11 & 1 & & 26 & 60 & 501 & . & 1 & . & . & Millet commun \\
\hline Panicum miliaceum & min. & . & . & 244 & . & . & . & . & . & . & . & Millet commun \\
\hline Secale cereale & carb. & . & . & & . & & . & & 1 & 2 & & Seigle \\
\hline Cerealia & carb. & . & 2 & . & $3+3,6 \mathrm{ml}$ & . & 1 & . & 8 & 1 & . & Céréales \\
\hline Cerealia & imb. & . & 26 & . & . & . & . & . & . & . & . & Céréales \\
\hline & & & & & & & & 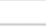 & & & 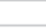 & \\
\hline Vannes & & & & & & & & & & & 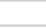 & \\
\hline Avena sativa, base épillet & imb. & 7 & . & . & . & . & . & . & . & . & . & Avoine cultivée \\
\hline Avena, base épillet & imb. & 3 & . & . & . & . & . & . & . & . & . & Avoine \\
\hline Hordeum, rachis & imb. & 1 & . & . & . & . & . & . & . & . & . & Orge \\
\hline Hordeum, rachis & carb. & 1 & & . & . & . & . & . & . & 16 & . & Orge \\
\hline Triticum dicoccon, base épillet & imb. & . & 296 & . & . & . & . & . & . & 3 & . & Amidonnier \\
\hline Triticum dicoccon, base épillet & carb. & . & 8 & . & . & . & . & . & . & 10 & . & Amidonnier \\
\hline Triticum dicoccon, base glume & imb. & 18 & 1001 & . & . & . & . & . & . & 23 & . & Amidonnier \\
\hline Triticum dicoccon, base glume & carb. & . & 24 & . & . & . & . & . & . & 43 &. & Amidonnier \\
\hline Triticum spelta, base épillet & imb. & . & 311 & . & . & . & . & . & . & 8 & . & Epeautre \\
\hline Triticum spelta, base épillet & carb. & 2 & 2 & . & . & . & . & . & . & . & . & Epeautre \\
\hline Triticum spelta, base glume & imb. & 10 & 1377 & . & . & . & . & . & . & 18 & . & Epeautre \\
\hline Triticum spelta, base glume & carb. & 20 & . & . & . & . & . & . & . & 17 & . & Epeautre \\
\hline T. dicoccon/spelta, base épillet & imb. & . & . & . & . & . & . & . & . & 7 & . & Amidonnier/Epeautre \\
\hline T. dicoccon/spelta, base glume & imb. & . & . & . & . & . & . & . & . & 24 & . & Amidonnier/Epeautre \\
\hline Triticum, base épillet & imb. & . & 73 & . & . & . & . & . & . & . & . & Blé \\
\hline Triticum, base glume & imb. & 3 & . & . & . & . & . &. & . & . & . & Blé \\
\hline Triticum, base glume & carb. & 1 & 117 & . & . & . & . & . & . & . & . & Blé \\
\hline Triticum, rachis & carb. & 5 & . & . & . & . & . & . & . & 6 & . & Blé \\
\hline Panicum miliaceum, épillet & imb. & 167 & 486 & . & . & & . & & & 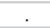 & & Millet commun \\
\hline Panicum miliaceum, glumelle & imb. & 1445 & 6856 & . & . & . & . &. & . & 36 &. & Millet commun \\
\hline Panicum miliaceum, glumelle & min. & . & . & 6 & . & . & . & . & . & . & . & Millet commun \\
\hline Panicum miliaceum, base palea & imb. & 1654 & 22125 & . & . & . & . & . & . & . & . & Millet commun \\
\hline Panicum miliaceum, fgt glumelle & imb. & 1135 & 8532 & . & . & . & . & . & . & . & . & Millet commun \\
\hline Secale cereale, rachis & carb. & . & . & . & . & . & . & . & . & 3 & . & Seigle \\
\hline Cerealia/Poaceae, nœud & imb. & 103 & 12 & . & . & . & . & . & . & . & . & Céréales/Graminées \\
\hline Cerealia/Poaceae, nœud & carb. & 35 & . & . & . & . & . & . & . & . & . & Céréales/Graminées \\
\hline Cerealia/Poaceae, fgt chaume & imb. & 40 & 17 & . & . & . & . &. & . & 8 &. & Céréales/Graminées \\
\hline Cerealia/Poaceae, fgt chaume/paille & carb. & . & . & . & . & . & * & . & . & 14 & . & Céréales/Graminées \\
\hline & & & & & & & & & & & 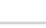 & \\
\hline Oléagineuses & & & & & & & & & & & & \\
\hline Linum usitatissimum, graine & imb. & . & 1 & . & . & . & . & . & . & 32 & . & Lin cultivé \\
\hline Linum usitatissimum, graine & carb. & . & . & . & . & . & . & . & . & 1 & . & Lin cultivé \\
\hline Linum usitatissimum, fgt capsule & imb. & 2 & 6 & . & . & . & . & . & . & 223 & . & Lin cultivé \\
\hline Linum usitatissimum, fgt capsule & carb. & . & . & . & . & . & . &. & . & 1 & . & Lin cultivé \\
\hline Papaver somniferum & imb. & 6 & 4 & . & . & . & . & . & . & . & . & Pavot somnifère \\
\hline Papaver somniferum & min. & . & . & 94 & . & . & . & . & . & . & . & Pavot somnifêre \\
\hline Camelina sativa agg. & $\min$. & . & . & 46 & . & . & . &. & . & . & . & Caméline \\
\hline Brassica/Sinapis & min. & . & . & 82 & . & . & . & . & . & . & . & Chou/Moutarde \\
\hline & & & & & & & & & & & & \\
\hline Légumineuses & & & & & & & & & & & & \\
\hline cf. Cicer arietinum & carb. & . & . & . & . & 2 & . & & & . & & cf. Pois chiche \\
\hline Lathyrus & carb. & . & . & . & . & . & 100 & . & . & . & . & Gesse \\
\hline Lens culinaris & min. & . & . & 35 & . & . & . & . & . & . & . & Lentille cultivée \\
\hline Lens culinaris & carb. & . & . & . & 82 & 293 & . & . & 142 & . & . & Lentille cultivée \\
\hline Pisum sativum & carb. & . & . & . & . & 115 & 98 &. & 322 & . & . & Pois cultivé \\
\hline Vicia faba & carb. & . & . & . & 94 & 7 & 10 & . & 103 & . & . & Féverole \\
\hline Vicia sativa agg. & carb. & . & . & 11 & . & . & . & . & . & . & . & Vesce cultivée \\
\hline Fabaceae & carb. & . & . & . & 1 & . & . & . & 1 & . & . & Légumineuses \\
\hline & & & & & & & & & & & & \\
\hline Aromates/légumes/épices & & & & & & & & & & & & \\
\hline Apium graveolens & imb. & 13 & 1 & . & . &. & . & . & . & 4 & . & Céleri \\
\hline Apium graveolens & min. & . & . & 1541 & . & . & . & . & . & . & . & Céleri \\
\hline Apium graveolens & carb. & . & . & . & . & . & . & . & 2 & . & . & Céleri \\
\hline Anethum graveolens & imb. & 1 & . & 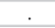 & . & . & . & . & . & 1 & . & Aneth \\
\hline Anethum graveolens & min. & . & . & 38 & . & . & . & . & . & . & . & Aneth \\
\hline Mentha/Origanum vulgare & min. & . & . & 29 & . & . & . & . & . & . & . & Menthe/Origan vulgaire \\
\hline
\end{tabular}




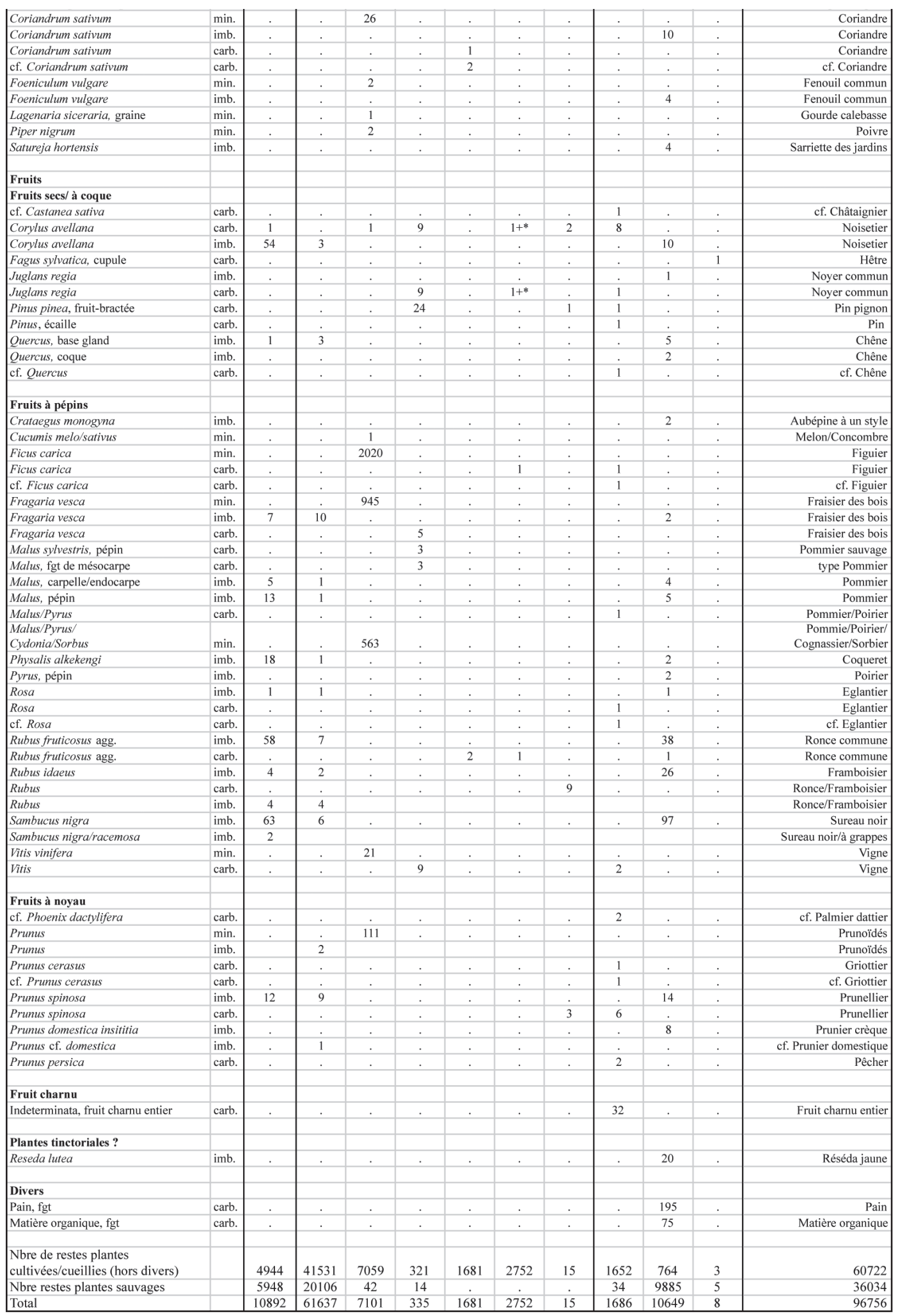

Abréviations : LTf : La Tène finale ; HtE : Haut-Empire ; BsE : Bas-Empire ;

$\mathrm{N}$ : nécropole; $\mathrm{Hr}$ : habitat rural : Civ : civitas ;

$\mathrm{p}$ : puits ; $f$ : fosse ; 1 : latrine ; inc : incinération

$\mathrm{c}$ : carbonisé ; $\mathrm{i}$ : imbibé ; $\mathrm{m}$ : minéralisé $;$ c f: détermination taxonomique incertaine
Tableau 3 : Limousin : plantes cultivées et cueillies de La Tène finale et de l'époque gallo-romaine.

Table 3: Limousin: cultivated and gathered plants of Late Celtic and gallo-Roman period. 
2000) (fig. 1 et tableau 1). Provenant de deux puits, le matériel végétal est, pour l'essentiel, conservé sous forme imbibée. Le spectre des plantes cultivées et utilitaires est formé de 4944 éléments. Il est principalement composé de vannes de céréales, de quelques oléagineuses, aromates et fruitiers (tableau 3). Les céréales mises en évidence sont constituées par le millet commun (Panicum miliaceum), attesté par de très nombreuses glumelles, puis par l'épeautre (Triticum spelta), l'amidonnier ( $T$. dicoccon), l'orge vêtue (Hordeum vulgare) et l'avoine cultivée (Avena sativa). Un grain carbonisé permet également d'attester la présence du blé nu (Triticum aestivum/durum). Du point de vue taxonomique, ce spectre est proche de celui des régions septentrionales françaises avec la mise en évidence des mêmes espèces à l'exception de l'engrain et de l'orge nue (Matterne, 2001; Lepetz et al., 2002; Malrain et al., 2002; Bakels, 2005). La présence de l'avoine cultivée est à souligner car la diffusion de l'espèce en France ne semble débuter qu'au second âge du Fer (Zech-Matterne et al., 2009). Pour le Limousin, l'importance relative des céréales est difficile à établir du fait de cette seule analyse. La représentativité de ces plantes peut, en effet, varier fortement selon le mode de conservation et les types de restes. Ainsi, l'orge et les différents blés peuvent être très diversement attestés selon que l'on considère les grains ou les résidus de vannage. C'est le cas, par exemple, des sites du second âge du Fer du Sud-Ouest de l'Allemagne dans lesquels l'épeautre est surtout déterminé sous forme de vannes alors que l'orge vêtue l'est essentiellement sous forme de grains (Rösch, 2006a). Cette apparente opposition peut résulter de modes de récolte, de stockage ou d'utilisation entre les céréales. Si un éventuel effet taphonomique peut être envisagé pour expliquer la meilleure conservation des bases d'épillets et de glumes d'épeautre par rapport aux rachis d'orge du fait de leur meilleure résistance à la carbonisation, cet argument ne peut être retenu pour expliquer la différence de représentation entre les grains d'épeautre et d'orge. La rareté des rachis d'orge dans les puits de SaintGence ne doit donc pas être interprétée comme le résultat du rôle secondaire de cette céréale en Limousin, les contextes étudiés n’ayant pas été favorables à la conservation des grains carbonisés.

Aucune légumineuse n’a été découverte. Cette absence n'est pas une surprise dans la mesure où, dans ces puits, le matériel carpologique est conservé à 98,7\% sous forme imbibée, un mode de conservation défavorable aux légumineuses (Green, 1979). Celles-ci sont pourtant bien représentées au second âge du Fer en France septentrionale et méridionale (Bakels, 1999; Matterne, 2001; Ruas et Marinval, 1991). Ces plantes jouent un rôle alimentaire important par leur apport en protéines. Leur absence en
Limousin est sans doute imputable à la nature de l'assemblage carpologique plutôt qu'à celle d'une consommation.

Seules deux oléagineuses ont été mises en evidence : le lin cultivé (Linum usitatissimum) par deux fragments de capsules et le pavot somnifère (Papaver somniferum) par six graines. Il en va de même pour les aromates pour lesquels seuls le céleri (Apium graveolens) et l'aneth (Anethum graveolens) ont été déterminés (tableau 3). Ces plantes appartiennent de longue date aux corpus des plantes cultivées et alimentaires. Les premières sont mentionnées en France depuis le Néolithique (Ruas et Marinval, 1991), les secondes sont attestées dès cette période en Suisse dans des stations lacustres (Jacomet, 1988; Brombacher, 1997), puis, de manière plus régulière, à partir de l'âge du Fer, en France comme en Allemagne (Wiethold, 1998; Matterne, 2001 ; Rösch et al., 2008).

Divers fruitiers ont éventuellement pu faire l'objet d'une collecte comme le noisetier (Corylus avellana), le chêne (Quercus), le hêtre (Fagus sylvatica), le prunellier (Prunus spinosa), le pommier (Malus), l'églantier (Rosa), le fraisier des bois (Fragaria vesca), le framboisier (Rubus idaeus), la ronce commune (Rubus fruticosus agg.) et le sureau noir (Sambucus nigra). Ces espèces spontanées en Limousin sont des composants naturels des formations forestières, des haies ou des landes. La présence du framboisier est à noter du fait de sa répartition actuelle en Limousin, limitée à la Montagne limousine et aux reliefs attenants. Mais au cours des deux derniers millénaires, sa répartition a pu varier car l'extension des milieux boisés a été largement modifiée sous les effets conjugués de l'impact anthropique et des modifications climatiques. Cette époque a en effet été marquée par des crises alluviales, signe d'un climat plus froid et humide ayant pu favoriser les massifs forestiers (Allée et al., 1997; Allée et Diot, 1996, 1997).

Des études sédimentologiques de banquettes agricoles fossilisées dans le paysage, associées à des datations ${ }^{14} \mathrm{C}$, ont permis de montrer qu'il existait à cette époque, une stabilisation du parcellaire de culture sur les meilleurs terroirs (Bernard-Allée et Valadas, 1992, 1993; Allée et al., 1996). Des amendements de terres ont également été mis en évidence lors de fouilles comme celles des Thermes à Limoges où un sol agricole gaulois a été découvert sous une voie de circulation. Il était constitué d'un apport de matières organiques, de calcium et de phosphate (ibid.).

\section{L'époque gallo-romaine}

\section{L'environnement végétal}

En Limousin, le début de la période gallo-romaine est marqué par une crise hydroclimatique qui se traduit par des 
phénomènes d'alluvionnement dans les vallées et les vallons (Allée et al., 1996, 1997). Les analyses palynologiques témoignent d'une anthropisation du milieu forestier supérieure à celle de l'âge du Fer. Les déboisements restent cependant modérés. Le hêtre (Fagus) est de plus en plus concurrencé par le chêne (Quercus) et le bouleau (Betula) (Lemée, 1949; Valadas, 1982), ce dernier colonisant sans doute diverses zones préalablement déboisées puis abandonnées. Cette évolution du couvert forestier est à la fois d'origine anthropique et climatique. Les données palynologiques montrent que les déforestations semblent avoir favorisé le chêne au détriment du hêtre (Allée et al., 1996, 1997; Allée et Diot, 1997). La diminution des pourcentages de cette espèce peut cependant être liée à une modification de son mode de traitement, l'émondage pour le fourrage entraînant une diminution de sa production pollinique (Jalut et al., 1982). A contrario, le chêne a pu être préservé pour son bois et ses fruits ainsi que pour son sous-bois qui autorise un pâturage sous forêt. Les variations de la production pollinique du hêtre sont cependant complexes. C'est une espèce qui s'avère très sensible à l'action anthropique ainsi qu'aux variations climatiques, même annuelles (Stebich et al., 2005). Les grains de pollen de Quercus, plus petits que ceux du hêtre, sont produits en plus grand nombre et mieux dispersés. Ils sont donc plus aptes à pénétrer tous les milieux, ce qui en fait, selon certains auteurs, une essence sur-représentée polliniquement (Guenet, in Miras, 2004). Pour d'autres, la question de la sur-représentation pollinique du chêne dépendrait de plusieurs facteurs et varierait selon les espèces, les techniques d'échantillonnage et les modes de transcription des données (Heim, 1970).

Cette prédominance du chêne sur le hêtre est perceptible dans les analyses anthracologiques (Fredon, 1985, 1995; Petit et Fredon, 2001; Petit, in Toledo i Mur et al., 2004). Les charbons de bois de chêne deviennent prédominants à l'époque gallo-romaine. Par rapport à la période précédente, ce renversement de tendance dans le choix des essences forestières utilisées comme combustible est interprété comme une surexploitation de la chênaie-hêtraie originelle dès la période protohistorique. Il aurait favorisé le développement des chênaies. Celles-ci sont plus rapides à se régénérer en milieu ouvert que les hêtraies. Cette ouverture des milieux serait confirmée, selon certains auteurs, par l'augmentation de la largeur moyenne des cernes du chêne entre l'âge du Fer et l'époque gallo-romaine (Petit, in Toledo i Mur et al., 2004).

\section{Les sites}

Neuf analyses carpologiques sont disponibles pour l'ensemble de la période. Six proviennent de nécropoles à incinérations (Marinval, 1991, 1993, 2001 ; Roger, 1995;
Toledo i Mur, 2004). Quatre d'entre elles sont datées du haut-Empire ( ${ }^{\mathrm{er}}-\mathrm{II}^{\mathrm{e}}$ siècles) : Bessines-sur-Gartempes, Mercoeur, Veyrac et Saint-Germain-les-Vergnes et deux de la transition Haut/Bas-Empire : Pontarion et SaintMaurice-la Souterraine (fig. 1, tableau 1 et 3). Les tombes ont livré un nombre de restes et de taxons très variable. La nécropole la plus riche est celle des Sagnes à Pontarion avec plus de 22 taxons et plus de 2700 restes carbonisés, ce qui en fait, à ce jour, la nécropole offrant la plus large gamme de végétaux (Marinval, 2001).

Les trois autres occupations sont constituées par le site proto-urbain du Patureau à Saint-Gence (Haute-Vienne), l'agglomération urbaine d'Augustoritum, l'antique Limoges (Haute-Vienne) et l'habitat rural de Lavaud à Gouzon (Creuse). Les deux premières sont datées du $\mathrm{I}^{\text {er }}$ siècle de notre ère, la troisième de la transition $\mathrm{II}^{\mathrm{e}} / \mathrm{III}^{\mathrm{e}}$ siècle. À SaintGence et à Gouzon, les analyses carpologiques sont issues de puits qui ont livré respectivement 61637 et 10649 restes, conservés sous forme imbibée à 99,9\% et à 98,6\% (tableau 2). À Limoges, les structures étudiées se composent de cinq fosses, dont quatre sont localisées à proximité d'un vaste bâtiment ceinturé d'un talus et d'une palissade identifiés comme un sanctuaire. Celles-ci ont livré un abondant matériel céramique et faunique et, à leur base, des restes carpologiques minéralisés (Maniquet [dir.], 1999). Ces comblements ont également fait l'objet d'analyses palynologiques (M.-F. Diot, inédit) et de spectrochimie (Pepe, inédit). Des larves d'insectes (arthropodes) minéralisées, en quantité variable selon les structures, ont été observées, ainsi que des concrétions pouvant s'apparenter à des «nodules sphéroïdes " (Ruas, 1995), mais aucun parasite intestinal n’a été repéré (com. orale M.-F. Diot). Deux hypothèses ont été émises concernant la fonction de ces fosses : comme dépotoirs, en ayant recueilli les rejets de repas cultuels ou rituels ou de sacrifices d'animaux comme le suggère la présence de viscères mises en évidence par la spectrochimie (Maniquet [dir.], 1999); excrémentielle, en ayant servi de latrines. Cette seconde hypothèse est suggérée par plusieurs indicateurs : la minéralisation des restes végétaux dont le mode de conservation peut résulter de taux élevés en phosphates ou en calcium (Green, 1979; Carruthers, 1991), le spectre carpologique qui est constitué essentiellement de fruits et de graines de plantes alimentaires, composé de semences de petits modules, comme de nombreux pépins, de graines d'oléagineuses et de semences d'aromates (Knörzer, 1984; Ruas, 1995). Parmi les autres critères figurent également les larves d'arthropodes considérées comme des indicateurs directs de sédimentation par les excréments (Karg, 1996), ainsi que les résultats d'analyses de spectrochimie qui rendent compte de forts taux de coprostanol et de cholane-24- 
oïque, le premier étant caractéristique d'une origine fécale, le second de viscères humaines ou animales. La présence de ces résidus intestinaux est ambiguë dans la mesure où leur origine animale est incertaine. De plus, ceux-ci peuvent résulter de rebus d'abattage sans avoir fait pour autant l'objet d'offrandes. L'absence de parasites intestinaux, considérés également comme des indicateurs directs de comblement de type latrine (Greig, 1983; Hellwig, 1997; Jacomet et Kreuz, 1999), va cependant à l'encontre de l'hypothèse de cette fonction. D'autres arguments peuvent être avancés comme une possible minéralisation des restes végétaux au contact des viscères.

\section{Productions et collectes végétales}

Les données carpologiques de cette période, plus nombreuses qu'à l'époque précédente, témoignent d'un spectre plus important et diversifié. Celui-ci bénéficie d'un corpus plus étoffé de sites et de structures ainsi que de modes de conservation plus variés des restes végétaux. Toutefois, peu de données proviennent de sites d'habitats, l'essentiel étant issu des nécropoles à incinérations.

\section{Céréales, légumineuses, oléagineuses}

Les céréales sont attestées sous la forme de grains et de vannes et par trois modes de fossilisation. Neuf espèces ont été identifiées. Leur occurrence varie selon le nombre de sites ou de structures (fig. 3 et 4). Ainsi, dans le premier cas, l'orge vêtue (Hordeum vulgare) devance le millet commun (Panicum miliaceum), l'avoine (Avena sp. et Avena sativa), l'amidonnier (Triticum dicoccon), le blé nu (T. aestivum/durum), l'épeautre (Triticum spelta), le seigle (Secale cereale), l'orge nue (Hordeum vulgare var. nudum) et l'engrain (Triticum monococcum). Dans le second cas, l'amidonnier arrive en deuxième position derrière l'orge vêtue, mais devant le blé nu, le millet commun et l'avoine. L'épeautre, le seigle, l'orge nue et l'engrain conservent les mêmes places. En termes d'abondance, c'est-à-dire de nombre de fois qu'une espèce domine sur un site, l'orge vêtue, l'amidonnier et le millet commun forment le trio de tête tout type de restes et modes de conservation confondus. Ils occupent chacun, deux fois, la première place (tableau 3). L'épeautre, le blé nu puis l'avoine suivent sans jamais prévaloir. La nécropole des Châtaigneraies à SaintGermain-les-Vergnes apparaît comme un cas à part dans la mesure où l'orge nue semble prédominer. Toutefois, le nombre de grains d'orge vêtue est exprimé par un mode volumétrique ne permettant pas une comparaison des deux types d'informations. Quelle que soit la place occupée par l'orge nue sur ce site, sa présence est à souligner car cette espèce disparaît des spectres carpologiques à compter du
$\mathrm{V}^{\mathrm{e}}$ siècle avant notre ère dans le nord de la France (Bakels, 1984, 1991, 1999; Matterne, 2001; Lepetz et al., 2002) et vers le $1^{\text {er }}$ age du $\mathrm{Fer}^{3}$ dans le sud (Marinval, 1988; Ruas et Marinval, 1991). Bien que beaucoup plus discret parmi les restes, l'engrain est attesté sur la nécropole des Sagnes à Pontarion. Il n'est toutefois mentionné que par un seul exemplaire pour un total de plus de 1000 grains. La structure dans laquelle a été identifié cet unique élément comprend une vingtaine de grains d'orge vêtue et à peu près autant d'amidonnier (Marinval, 2001). L'engrain n'apparaît donc que de façon relictuelle sur ce site.

Bien que l'avoine soit mentionnée sur cinq occupations sous forme de grain nu, l'avoine cultivée ne figure que furtivement parmi les restes. Elle est seulement attestée sur un site, la nécropole de Veyrac. Le seigle est présent dans deux occupations des II $^{\mathrm{e}}$-III ${ }^{\mathrm{e}}$ siècles toutes deux localisées en Creuse, la nécrople des Sagnes à Pontarion et l'habitat rural de Lavaud à Gouzon. Au regard du nombre d'éléments identifiés, son importance semble modeste et ne permet pas de certifier sa culture. Plusieurs analyses palynologiques de sites archéologiques, de tourbières ou de bas-fonds tourbeux du Limousin ont également livré des occurrences polliniques, mais de façon sporadique. Les analyses de l'ancien étang de la villa des Cars en Montagne limousine sont les seules à avoir pu mettre en évidence une culture de cette céréale aux alentours du $\mathrm{II}^{\mathrm{e}}$ siècle de notre ère (Miras, 2004; Diot et al., 2006).

L'introduction du sarrasin ou blé noir (Fagopyrum esculentum) en Limousin à l'époque gallo-romaine est également discutée. Aucun akène n’a été déterminé. Seuls quelques grains de pollen ont été identifiés dans la sépulture du début de l'époque augustéenne de Saint-Augustin en Corrèze (Marambat, in Dussot et al., 1992), à l'ancien étang des Cars (Corrèze) (Miras, 2004; Diot et al., 2006), à la Védrenne (Creuse) (Freytet et al., 1989) et dans un bas-fond tourbeux au Martoulet (Haute-Vienne) (Allée et al., 1997). Dans les diagrammes polliniques du Limousin, cette espèce apparaît donc plus tardivement que dans d'autres régions françaises, comme dans l'Ouest où quelques grains de pollens ont été identifiés dès le Néolithique (Marguerie, 1992; Cyprien et al., 2004; Visset et al., 2005), ou encore sur le littoral catalan où plusieurs occurences ont été mises en évidence à l'âge du Bronze (Riera i Mora, 1994). Dans le Massif armoricain, en Mayenne et en Campine belge, des tourbières de l'âge du fer ont également livré des pollens (Marguerie, 1992; Barbier, 1999; Munaut, 1967).

Les légumineuses n'ont été identifiées que sur cinq sites soit quatre nécropoles et la Civitas de Limoges. Les

3. Soit 700 avant notre ère. 


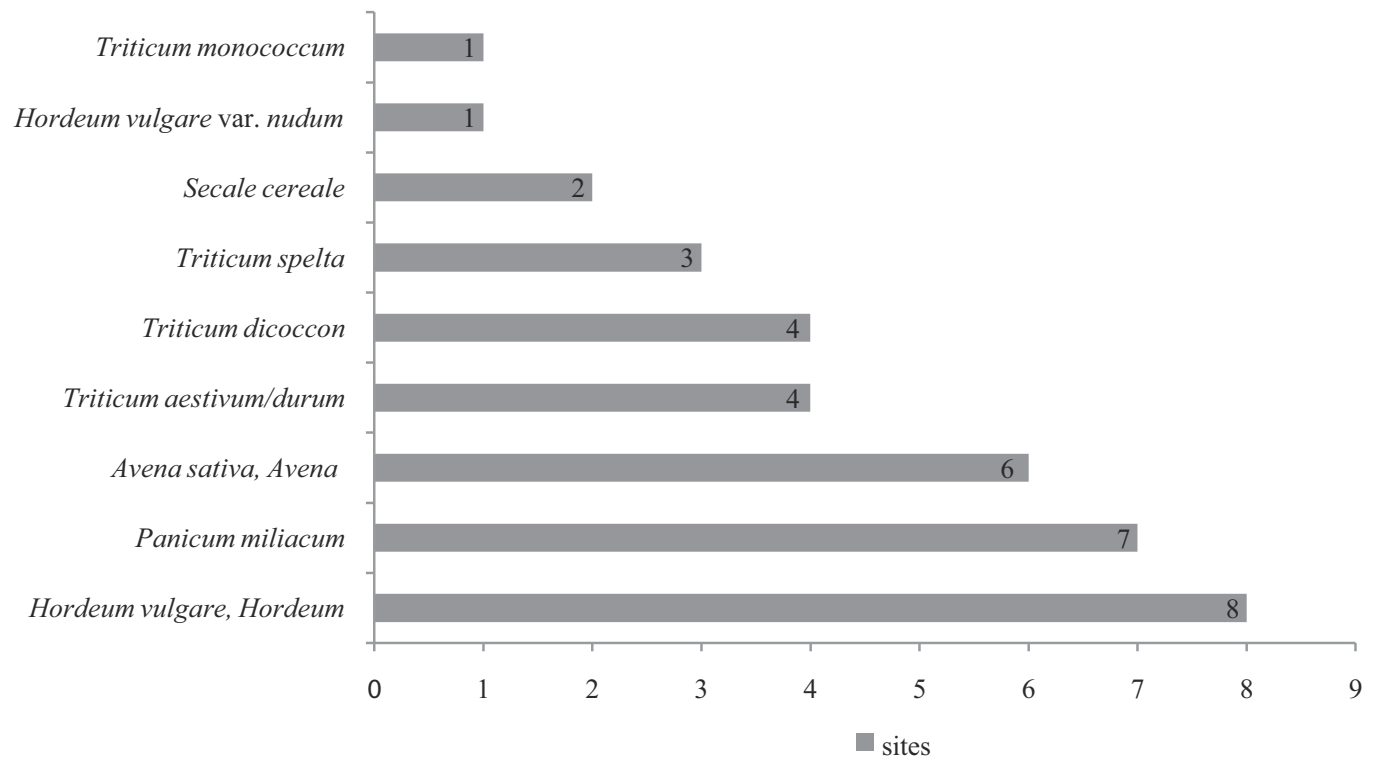

Figure 3 : Occurrence des céréales par site pour l'époque gallo-romaine (total sites : 9). Figure 3: Occurrence of cereals per site during the Gallo-Roman period (nb of sites: 9).

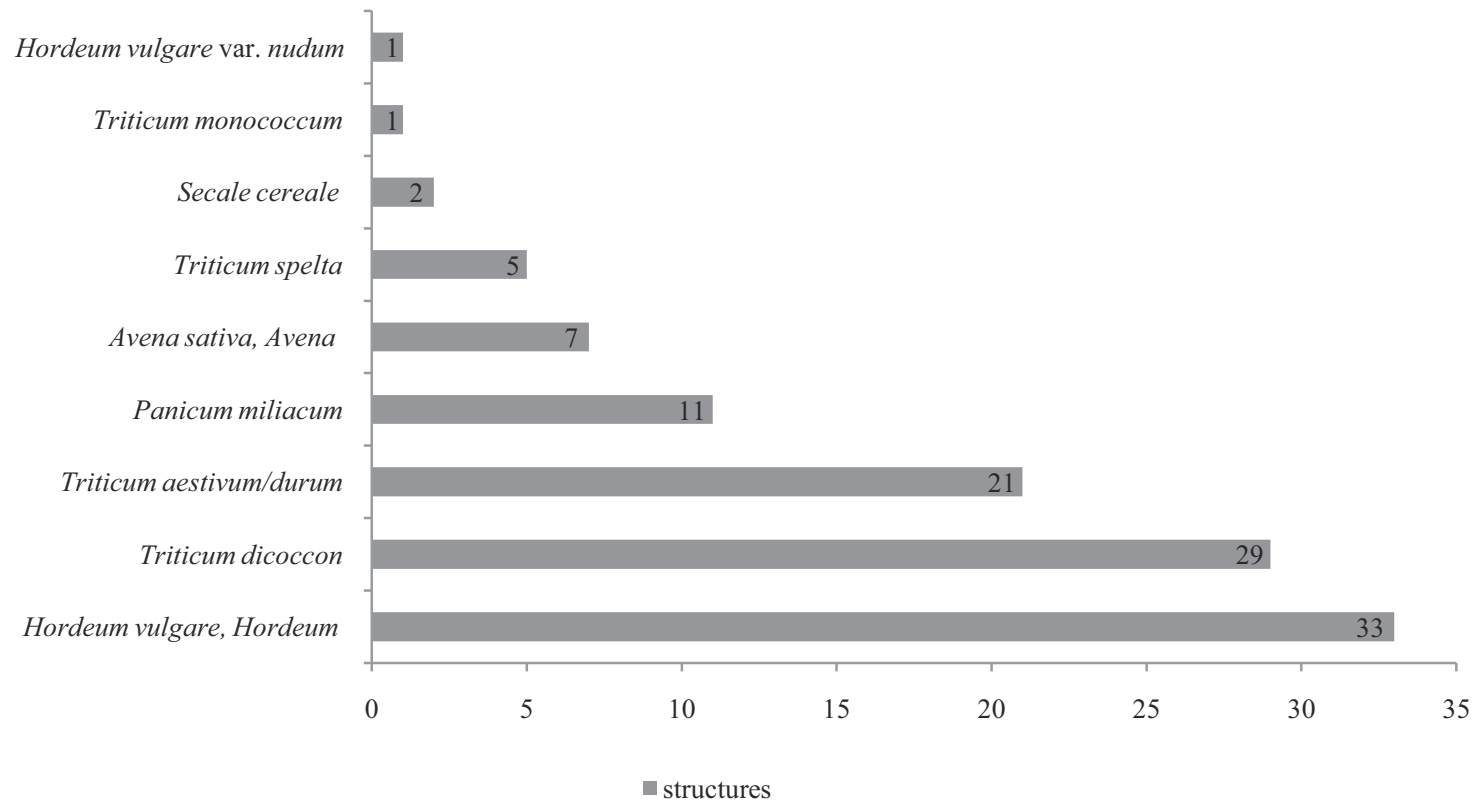

Figure 4 : Occurrence des céréales par structure pour l'époque gallo-romaine (total structures : 89).

Figure 4: Occurrence of cereals per structure during the Gallo-Roman period (nb of structures : 89).

nécropoles sont celles de Saint-Germain-les-Vergnes, Veyrac, Bessines-sur-Gartempes et Pontarion. Quatre des cinq sites concernent le haut-Empire, la nécropole des Sagnes à Pontarion étant la seule à apporter des informations pour les $\mathrm{II}^{\mathrm{e}}-\mathrm{III}^{\mathrm{e}}$ siècles. Le spectre des légumineuses se compose de sept taxons, dont quatre espèces : la lentille cultivée (Lens culinaris), le pois cultivé (Pisum sativum), la féverole (Vicia faba), la vesce cultivée (Vicia sativa agg.).
Les trois autres sont formés par la gesse (Lathyrus), des légumineuses indéterminées et, avec incertitude, par le pois chiche (cf. Cicer arietinum). La féverole et la lentille cultivée sont mentionnées sur quatre occupations : des nécropoles uniquement pour la première, le site urbain de Limoges et trois nécropoles pour la seconde. Le pois cultivé est attesté sur trois sites funéraires. En termes d'abondance, la féverole prédomine sur une nécropole, la 
lentille cultivée sur une autre et le pois cultivé sur une troisème. La lentille cultivée est également représentée à Limoges, en contexte urbain. La nécropole de Veyrac se distingue des autres sites par une prépondérance de graines de gesse (Lathyrus). Deux espèces potentielles, difficiles à distinguer, sont concernées : la gesse cultivée et la gesse chiche (Marinval, 1986). Cette dernière a été identifié dans le Nord de la France à l'époque gallo-romaine (Lepetz et al., 2002; Matterne, 2001, 2003). La première, beaucoup moins fréquente, est principalement attestée autour du bassin méditerranéen. La vesce cultivée, dont les déterminations sont peu courantes à l'époque gallo-romaine, n’a été mise en évidence qu'à Limoges. Elle provient du comblement d'une fosse riche en grains de céréales. Cette espèce, connue dans le Centre-Est dès le $1^{\text {er }}$ âge du Fer (Wiethold, 1998, 1999), est mentionnée ponctuellement dans le Nord de la France à partir du $\mathrm{I}^{\mathrm{er}}$ siècle de notre ère (Matterne, 2003) et attestée également ponctuellement dès le Néolithique moyen en Méditerranée occidentale (Bouby et Léa, 2006).

Trois espèces composent le corpus des oléagineuses : le lin cultivé (Linum usitatissimum), le pavot somnifère (Papaver somniferum) et la caméline (Camelina sativa). À ces déterminations s'ajoute celle du chou ou de la moutarde (Brassical Sinapis). Ces données proviennent de l'occupation urbaine de Limoges, l'habitat proto-urbain de Saint-Gence-lePatureau et celui rural de Gouzon-Lavaud. Plusieurs modes de conservation sont attestés : l'imbibition et la carbonisation pour le lin, la minéralisation et l'imbibition pour le pavot, la minéralisation seulement pour les brassicacées. Seules deux espèces ont été identifiées sur deux occupations : le lin et le pavot. La première, attestée à la fois sous forme de graines et de fragments de fruits, est mentionnée à Limoges et à Gouzon, la seconde à Saint-Gence et à Limoges où la fossilisation minéralisée s'est révélée très favorable à sa conservation. La présence de caméline est à souligner. Sa diffusion en France s'effectue dès le Néolithique moyen (Bouby, 1998) et elle semble perdre de son importance à l'époque gallo-romaine (Marinval, 2005). Elle devient anecdotique dans le Nord de la France dès la fin du second âge du Fer (Matterne, 2001). À Limoges, les graines de caméline recueillies sont issues d'assemblages carpologiques composés essentiellement de plantes cultivées. Il est donc probable que cette espèce ait eu le même statut plutôt que celui d'une mauvaise herbe de culture.

Comme pour la période précédente, des analyses sédimentologiques ont permis de mettre en évidence une fixation des champs sur les meilleurs terroirs, notamment autour des villae. Elles rendent compte également d'un amendement des champs comme à Saint-Germain-les-Belles-Le Martoulet
(Haute-Vienne), Peyrilhac-la Boisserie (Haute-Vienne) et Chaumeil-Maurianges (Corrèze) (Allée et al., 1996; Allée et Diot, 1997; Desbordes et Valadas, 1998). Cette intensification des cultures peut avoir répondu à plusieurs nécessités : une augmentation de la pression démographique, une volonté de dégager des surplus, une bonification des sols au regard de l'espace disponible autour des implantations humaines (Van der Veen, 2005).

\section{Arboriculture et horticulture}

Par rapport à celle de la période précédente, la liste des fruits mis en évidence à l'époque gallo-romaine s'est largement étoffée du fait des différents modes de conservation et de la diversification des sites étudiés. Ainsi, une trentaine de plantes a été recensée dont 17 espèces. En dépit du nombre très variable de restes recueillis par site, tous en ont livré. Le noisetier (Corylus avellana) est l'espèce la plus fréquente avec 7 mentions suivi de la ronce commune (Rubus fruticosus agg.), du fraisier des bois (Fragaria vesca), du noyer commun (Juglans regia) et du prunellier (Prunus spinosa), puis du figuier (Ficus carica), du pin pignon (Pinus pinea), de l'églantier (Rosa), de la vigne (Vitis). D'autres ne sont attestés que sur un ou deux sites : le chêne (Quercus), le framboisier (Rubus idaeus), le sureau noir (Sambucus nigra), l'aubépine à un style (Crataegus monogyna), le concombre/ melon (Cucumis melo/sativus), le hêtre (Fagus), le griottier (Prunus cerasus), le pêcher (Prunus persica), le prunier crèque (Prunus domestica insititia), le pommier (Malus), le poirier (Pyrus). À cela s'ajoutent quelques taxons identifiés de manière incertaine : le châtaignier (cf. Castanea sativa), le palmier dattier (cf. Phoenix dactylifera) et le prunier domestique (Prunus cf. domestica). La représentation carpologique de ces fruits est très variable, allant de 1 à 2020 restes pour le figuier. Celui-ci est en effet représenté par de nombreux pépins à Limoges, tout comme le fraisier des bois, les pruniers et les pommier/poirier/sorbier. La minéralisation de ces fruits et graines n'a pas toujours permis des déterminations spécifiques. C'est également de ce site que provient l'une des rares mentions de pépins de vigne, les deux autres étant issues des nécropoles à incinérations des Châtaigneraies à Saint-Germain-les-Vergnes et des Sagnes à Pontarion. La rareté de ces déterminations est à souligner, dans la mesure où elle égale celles de deux plantes exotiques d'origine méditerranéenne : le pin pignon et le figuier. La vigne est aussi absente des données anthracologiques publiées à ce jour pour l'époque galloromaine (Fredon, 1985, 1995; Petit, in Toledo i Mur, 2004). Elle apparaît de manière très sporadique dans les analyses palynologiques. Cette discrétion peut résulter de la faible dispersion de son pollen entraînant des taux de 
représentation peu élevés (Cour et al., 1973; Meiffren, 1984), l'espèce étant autogame. La rareté de ces diverses attestations pose la question de l'existence d'une viticulture en Limousin, d'autant qu'aucune trace de plantations de vigne n'a été, jusqu'à présent, mise au jour. Aussi, la vigne a pu faire l'objet d'importations sous forme de fruits séchés, tout comme ceux du pin pignon et du figuier. Toutes ces espèces produisent des fruits qui se conservent très bien séchés, ce qui les rend aisément commercialisables. Des procédés autres que la dessication ont même parfois été utilisés par les Romains pour conserver le raisin (André, 1961). Toutefois, le développement d'une viticulture a été mis en évidence dans des régions plus septentrionales que le Limousin, comme en pays ligérien ou dans le Val-d'Oise (Couderc et Provost, 1990; Lefevre, 1990; Toupet et al., 2003). On peut donc envisager un tel développement au moins à proximité de l'agglomération urbaine d'Augustoritum. En France septentrionale, l'hypothèse d'une acclimatation de certaines espèces méditerranéennes, tel que le pin pignon, a également été envisagée (Marinval et al., 2002), le climat de l'époque étant plus chaud et sec que de nos jours (Desprat et al., 2003).

Les contextes analysés ne se sont pas montrés favorables aux divers pruniers. Ainsi, le griottier (Prunus cerasus) n'est mentionné que sur la nécropole à incinérations des Sagnes à Pontarion, de même que le pêcher (Prunus persica). Le prunier domestique (Prunus cf. domestica) est attesté de manière incertaine dans le puits du Patureau à Saint-Gence et le prunier crèque (Prunus domestica ssp. insititia) dans celui de Lavaud à Gouzon. Aucune détermination de merisier (Prunus avium) n'est signalée. Cette situation résulte pour partie des noyaux de Prunus trouvés à Limoges dont la minéralisation n'a pas permis d'identification spécifique. Elle est également consécutive à la nature des contextes archéologiques constitués essentiellement d'incinérations. À l'exception de celles de la nécropole de Sagnes, elles ont livré peu de fruitiers. Il en va de même pour le site de Lavaud à Gouzon dont le spectre est essentiellement composé d'espèces indigènes, à l'exception du noyer.

Le châtaignier (Castanea sativa), espèce emblématique du Limousin, n’a été déterminé avec incertitude que sur la nécropole de Sagnes à Pontarion (Marinval, 2001). Il est attesté par des charbons de bois sur deux sites du haut-Empire : la Chapelle Montbrandaix et Châtenet-les-Dognons (HauteVienne) (Fredon, 1985, 1995). L'hypothèse d'une introduction plus ancienne a été envisagée sur la base d'analyses palynologiques effectuées dans deux fonds de vallons des bas-plateaux limousins au sud-est de Limoges montrant la présence de grains de pollen de Castanea dans des dépôts datés de l'âge du Fer (Allée et al., 1997). La présence de ces grains à la base d'un dépôt organo-minéral pourrait être liée à une contamination (Miras, 2004). L'indigénat de cette espèce en Limousin demeure donc hypothétique d'autant plus qu'e cette entité géographique n’a pas été retenue comme l'une des six macro-régions où le châtaignier aurait pu trouver des conditions favorables à sa survie au cours du dernier épisode glaciaire (Conedera et al., 2004).

Pour des raisons taphonomiques, les aromates et les légumes sont des plantes rarement mises en évidence. Sept espèces ont néanmoins été identifiées. Elles sont issues de cinq occupations : Saint-Gence-le Patureau, Limoges, Gouzon et les nécropoles des Ribières à Bessines-surGartempes et des Sagnes à Pontarion. Elles sont donc attestées à la fois en milieu urbain, rural et funéraire. Ce spectre résulte aussi de la diversité des modes de conservation du fait de leur mise en évidence sous forme imbibée, carbonisée et/ou minéralisée. Les espèces les plus fréquentes en termes de mentions et de nombre de restes sont successivement le céleri (Apium graveolens), l'aneth (Anethum graveolens), la coriandre (Coriandrum sativum) puis le fenouil commun (Foeniculum vulgare). La sarriette des jardins (Satureja hortensis), la gourde calebasse (Lagenaria siceraria) et le poivre (Piper nigrum) ne font l'objet que d'une seule mention (fig. 5). La première a été mise en évidence sous forme imbibée à Gouzon, les suivantes, sous forme minéralisée à Limoges. Attribuée par le mobilier céramique à la première moitié du ir siècle, l'attestation de la gourde calebasse et du poivre, est remarquable. En effet, de telles découvertes sont peu fréquentes et toutes plus tardives pour la gourde calebasse. C'est le cas à Mazières-en-Mauges en Maine-etLoire (Dietrich et Ruas, 1990; Ruas, 2000), à Longueil Sainte-Marie dans l'Oise (de Hingh, 1993, Marinval et al., 2002) et à Alésia-le-Belvédère en Côte-d'Or (Wiethold, 1998). Il en est de même pour les provinces romaines d'Europe centrale en particulier pour Hanau-am-Salisberg en Hesse (Kreuz, 1994/1995), à Dieburg (Göldner et Kreuz, 1999) et pour Lahr-Dinglingen dans le Baden-Württemberg (Rösch, 1998). Les découvertes de poivre dans la partie septentrionale de l'Empire romain sont encore plus rares. Les quelques identifications connues proviennent des camps militaires d'Oberaden à Bergkamen en Rhénanie-Westphalie (Allemagne) (Kučan, 1984, 1992) et de celui d'CEdenburg en Alsace (France) (Jacomet et Schibler, 2002). La rareté de ces identifications tient aussi au mode de consommation de cette épice qui, sous forme moulue ou broyée, ne laisse pas de traces. Cette espèce exotique originaire de l'Inde était très appréciée des Romains (André, 1961). Son origine en fait un produit d'importation, vraisemblablement destiné à une clientèle aisée. Elle a pu suivre, à partir des rives de la Méditerranée, les mêmes routes commerciales que celles de 


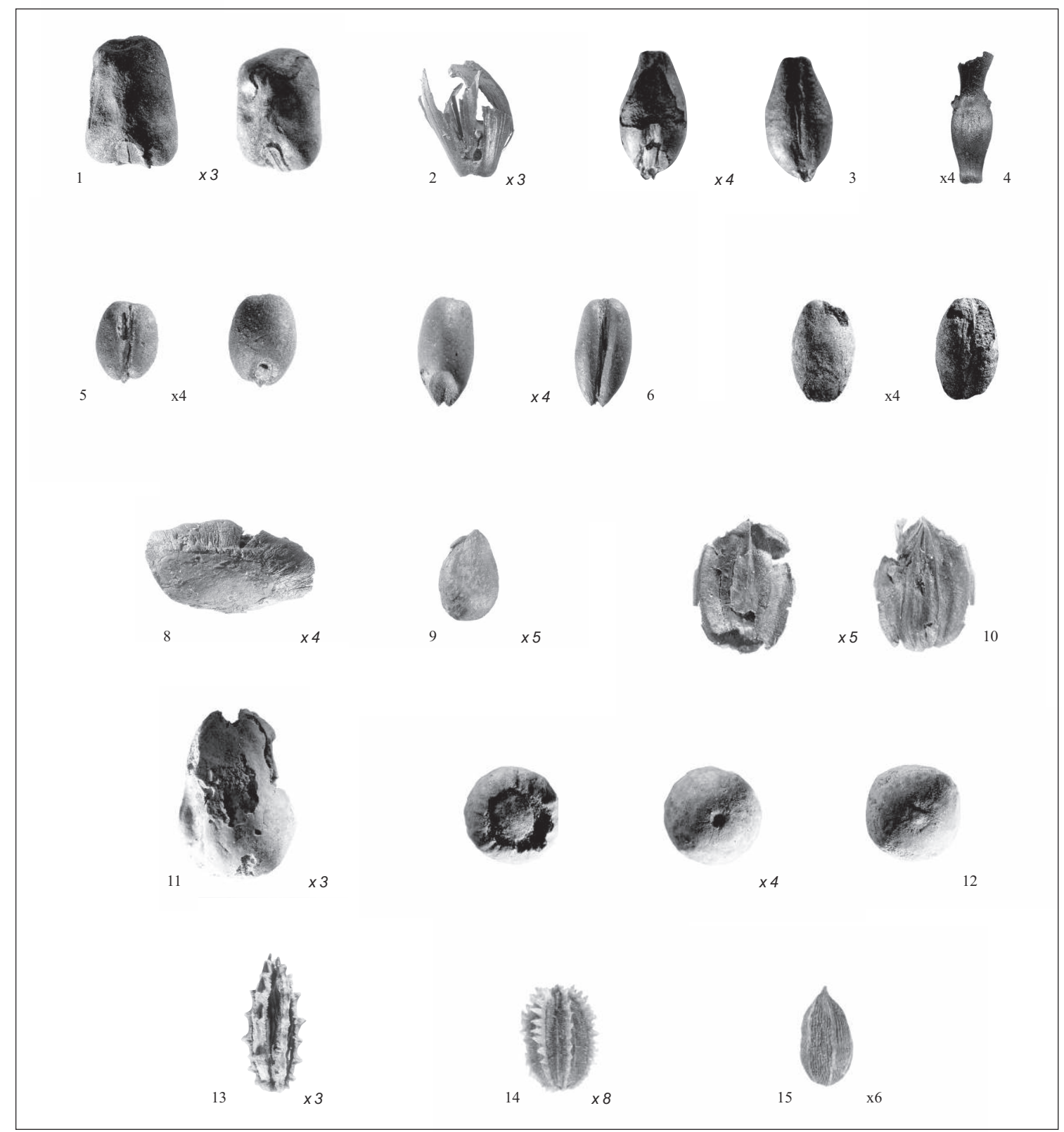

Figure 5 : (Voir planche couleur) Limousin : Plantes cultivées et sauvages : 1 :Féverole (Vicia faba), Limoges-Cnasea; 2 : Épeautre (Triticum spelta), Saint-Gence-Le Patureau; 3 : Orge vêtue (Hordeum vulgare), Limoges-CHR; 4 : Blé tendre (Triticum aestivum), Gouzon-Lavaud; 5 : Blé tendre/dur (Triticum aestivum/durum), Limoges-Cnasea; 6 : Seigle (Secale cereale), Limoges-Cnasea; 7 : Orge nue (Hordeum vulgare var. nudum), Aix-Le Grandcher; 8. Châtaignier (Castanea sativa), Limoges-Le Maupas; 9 : Mûrier noir (Morus nigra), Limoges-Le Maupas; 10 : Panais (Pastinaca sativa), Limoges-Le Maupas; 11 : Gourde calebasse (Lagenaria siceraria), Limoges-CHR; 12 : Poivre noir (Piper nigerum), Limoges-CHR; 13 : Caucalis à fruits plats (Caucalis platycarpos), Limoges-Le Maupas; 14 : Carotte sauvage (Daucus carota), Gouzon-Lavaud; 15 : Cigüe tachetée (Conium maculatum), Gouzon-Lavaud. (Photos: Anne Bouchette; DAO : Laurent Cordier, INRAP). Figure 5: (See colour plate) Limousin: Cultivated and wild plants: 1: Celtic bean (Vicia faba), Limoges-Cnasea; 2: Spelt (Triticum spelta), Saint-Gence-Le Patureau; 3: Hulled barley (Hordeum vulgare), Limoges-CH R; 4: Bread wheat (Triticum aestivum), Gouzon-Lavaud; 5: Nacked wheat (Triticum aestivum/durum), Limoges-Cnasea; 6: Rye (Secale cereale), Limoges-Cnasea; 7: Nacked barley (Hordeum vulgare var. nudum), Aix-Le Grandcher; 8: Sweet Chestnut (Castanea sativa), Limoges-Le Maupas; 9: Black Mulberry (Morus nigra), Limoges-Le Maupas; 10: Wild Parsnip (Pastinaca sativa), Limoges-Le Maupas; 11: Bottle-gourd (Lagenaria siceraria), Limoges-CHR; 12: Pepper (Piper nigerum), Limoges-CHR; 13: Small Bur-parsley (Caucalis platycarpos), Limoges-Le Maupas; 14: Carrot (Daucus carota), Gouzon-Lavaud; 15: Hemlock (Conium maculatum), Gouzon-Lavaud (Photos: Anne Bouchette; DAO: Laurent Cordier, INRAP). 
fruitiers qui ont pu faire aussi l'objet d'importations comme le pin pignon, le figuier, la gourde calebasse, etc., dans la mesure où leur acclimation en Limousin est incertaine. Plus en amont, le poivre a pu emprunter les mêmes routes commerciales que celles d'autres produits exotiques comme le coton, dont des fils ont été retrouvés insérés dans un amas de tissus et de cordelette de lin et de chanvre. Ces restes proviennent d'une incinération à Fontvieille sur la commune de Vareilles en Creuse, datée de la seconde moitié du II $^{\mathrm{e}}$ siècle (Lorquin et Moulhérat, 2001-2002; Dussot et al., 2002). Selon les auteurs, l'emploi de cette matière exotique ne pouvait résulter que d'une importation, probablement d'Inde, via le commerce égyptien. Mais une provenance nubienne ne peut être totalement écartée. Contrairement à ce que pourrait laisser supposer les découvertes faites à Limoges, celle de Fontvieille montre que les importations exotiques ne relevaient pas que du phénomène urbain.

À partir de la fin du III ${ }^{\mathrm{e}}$ siècle, les diagrammes polliniques indiquent une baisse de la pression anthropique sur les paysages végétaux qui se traduit par une reconquête forestière plus ou moins prononcée selon la localisation des gisements. De courte durée, elle paraît plus conséquente en Montagne limousine que sur les bas-plateaux.

Si les données archéologiques et archéobotaniques, plus abondantes qu'aux époques précédentes, permettent d'être plus prolixe en matière de reconstitution paléoenvironnementale, en particulier, en ce qui concerne les productions végétales, il est à souligner que ces dernières ne sont plus seulement le fait d'une exploitation locale, mais aussi de productions allochtones.

\section{Le Moyen Âge}

\section{Le haut Moyen Âge ( $V t^{e}-X^{e}$ siècles)}

Les données palynologiques disponibles pour cette époque montrent que le Limousin connaît une vague de défrichements attribuables à l'époque mérovingienne au moins en ce qui concerne les bas-plateaux (Allée et al., 1996, 1997). Cette ouverture du milieu s'inscrit dans une phase de crise alluviale qui suppose une détérioration climatique (Burnouf et al., 1997; Magny et al., 2003). Il semble que la Montagne limousine n'ait pas enregistré le même phénomène, peut-être à cause des conditions climatiques plus difficiles.

Les données carpologiques sont issues de deux occupations localisées à Limoges : celle du Maupas et celle du CNASEA. La première occupe un espace rural en périphérie des deux pôles urbains de la ville représentés, pour l'un, par la Cité avec la Cathédrale, pour l'autre par la motte du vicomte de Limoges et l'abbaye Saint-Martial. Les prélèvements proviennent du comblement d'un puits qui a été daté par le mobilier céramique des $\mathrm{VI}^{\mathrm{e}}$-VIII ${ }^{\mathrm{e}}$ siècles. Le second site est localisé à proximité du précédent. Il comprend trois fosses pour lesquelles une datation ${ }^{14} \mathrm{C}(690$ cal. AD-975 cal. AD) (1 sigma d'erreur) a été obtenue sur des paléosemences (Massan et al., 2002). Les quatres structures analysées ont fourni 47693 restes imbibés et carbonisés de plantes cultivées et cueillies pour un total de 58077 (tab. 4).

\section{Productions et collectes végétales}

L'assemblage carpologique des structures des deux occupations differe de manière importante. Celui du Maupas est composé à $98 \%$ de restes imbibés, celui du CNASEA à $100 \%$ de semences carbonisées. Le corpus des plantes déterminées se différencie donc par le mode de conservation. Le premier site a livré essentiellement des fruits, des aromates et des légumes; le second, des céréales et des légumineuses. L'information recueillie est donc disparate entre les deux sites. Les trois fosses du CNASEA ont livré des restes végétaux en quantités très variables. L'une d'elles contenait à sa base un niveau charbonneux riche en grains de céréale dont $96 \%$ de seigle (Secale cereale, fig. 5). Cette espèce domine également, en nombre de restes, dans l'une des deux autres structures. Concernant la troisième, la quantité de restes déterminés est trop faible pour rendre compte, en termes d'abondance, du spectre des céréales. Le seigle est également représenté dans le puits du Maupas dont la datation est un peu plus ancienne. Cette céréale est attestée par près d'une soixantaine de grains carbonisés et des fragments de rachis imbibés et carbonisés. Les autres espèces mises en évidence dans cette structure sont constituées par l'avoine cultivée (Avena sativa) avec trois bases de lemme. Près d'une quarantaine de caryopses d'avoine (Avena) a également été identifiée. Trois autres espèces ont été observées : le millet commun (Panicum miliaceum) sous forme de glumelles imbibées, le blé nu (Triticum aestivum/durum) par un grain et un fragment de rachis carbonisés, et l'épeautre (Triticum spelta), par un grain carbonisé. Excepté cette dernière, toutes les autres céréales figurent parmi les déterminations du CNASEA auxquelles s'ajoute l'orge vêtue (Hordeum vulgare). Ainsi, le spectre des plantes mises en évidence sur ces deux sites en contexte urbain ou péri-urbain, differre de façon assez importante de celui de l'époque gallo-romaine. La rareté des restes de millet commun, d'épeautre et l'absence d'amidonnier est à souligner. Ce dernier tend à disparaittre au cours du haut Moyen Âge, tant en France méridionale que septentrionale (Ruas, 1998, 2005; Bakels, 1999; Lepetz et al., 2002). L'importance prise par le seigle est indéniable. La place occupée par l'avoine cultivée, mentionnée sur les deux sites, demande à être précisée, celles du blé nu et de l'orge vêtue sont difficiles à définir. 


\begin{tabular}{|c|c|c|c|c|c|c|c|c|c|c|}
\hline $\mathrm{N}^{\circ}$ sites & & 10 & 11 & 12 & 13 & 14 & 14 & 12 & 15 & \\
\hline Chronologie & & HtMA & HtMA & MAC & MAC & MAC & BMA & BMA & BMA & \\
\hline Site & & $\mathrm{V}$ & $\mathrm{V}$ & $\mathrm{Hr}$ & V & $\mathrm{BC}$ & $\mathrm{BC}$ & $\mathrm{Hr}$ & $\mathrm{Ca}$ & \\
\hline Structures & & $\mathrm{p}$ & $\mathrm{f}$ & $\mathrm{f}$ & fé & f & f, sout & & no & \\
\hline Conservation & & $\mathrm{i}, \mathrm{c}$ & $\mathrm{c}$ & $\mathrm{c}$ & $\mathrm{i}$ & $\mathrm{c}$ & $\mathrm{i}, \mathrm{c}$ & $\mathrm{c}$ & c & \\
\hline Taxons & Etat & & & & & & & & & Nom français \\
\hline \multicolumn{11}{|l|}{ Céréales } \\
\hline \multicolumn{11}{|l|}{ Grains } \\
\hline Avena & carb. & 38 & 38 & 10966 & . & 1008 & 51 & 170 & 8 & Avoine \\
\hline Avena sativa & carb. & . & 1 & . & . & . & . & . & . & Avoine cultivée \\
\hline Avena strigosa & carb. & . & . & . & . & . & . & . & . & Avoine rude \\
\hline Hordeum vulgare & carb. & . & 11 & 11 & . & 21 & 3 & . & 4 & Orge polystique vêtue \\
\hline Hordeum vulgare var. nudum & carb. & . & . & 1 & . & . & . & . & . & Orge nue \\
\hline Hordeum & carb. & . & . & 2 & . & 2 & . & . & . & Orge \\
\hline Panicum miliaceum & carb. & . & 4 & . & . & 12 & 4 & . & 1 & Millet commun \\
\hline Triticum aestivum/durum & carb. & 1 & 106 & 1 & 1 & 271 & . & 6 & . & Blé tendre/dur \\
\hline Triticum dicoccon & carb. & . & . & . & . & 2 & . & . & . & Amidonnier \\
\hline Triticum spelta & carb. & 1 & . & . & . & . & . & . & . & Epeautre \\
\hline Secale cereale & carb. & 57 & 45088 & 115 & . & 14612 & 104 & 17 & . & Seigle \\
\hline Setaria italica & carb. & . & . & . & . & 1 & 7 & . & . & Millet des oiseaux \\
\hline Cerealia & carb. & 9 & 4 & 3 & . & 118 & 18 & 3 & 1 & Céréales \\
\hline Cerealia & imb. & 1 & . & . & . & . & . & . & . & Céréales \\
\hline \multirow{2}{*}{\multicolumn{11}{|c|}{ Vannes }} \\
\hline & & & & & & & & & & \\
\hline Avena, base épillet & carb. & 1 & . & . & . & . & . & 4 & . & Avoine \\
\hline Avena sativa, base épillet & carb. & 3 & . & . & . & 11 & . & . & . & Avoine cultivée \\
\hline Avena sativa, épillet & carb. & . & $\dot{.}$ & $\dot{.}$ & $\dot{.}$ & . & $\dot{i}$ & . & $\dot{.}$ & Avoine cultivée \\
\hline Avena sativa, base épillet & imb. & . & . & & . & . & 8 & . & . & Avoine cultivée \\
\hline Avena strigosa, épillet & carb. & . & . & 135 & . & . & . & . & . & Avoine rude \\
\hline Avena strigosa, base épillet & carb. & . & . & 185 & . & . & . & 6 & . & Avoine rude \\
\hline Avena cf. strigosa, base épillet & carb. & $\dot{.}$ & $\dot{.}$ & 1 & $\dot{.}$ & $\dot{.}$ & $\dot{.}$ & $* * *$ & $\dot{.}$ & cf. Avoine rude \\
\hline Avena, barbe & carb. & $\dot{.}$ & $\dot{.}$ & 166 & $\dot{.}$ & 1 & 1 & . & . & Avoine \\
\hline Hordeum, rachis & imb. & . & . & . & $\dot{.}$ & . & 1 & . & $\dot{.}$ & Orge \\
\hline Panicum miliaceum, gl./base & imb. & 3 & . & . & . & . & 441 & . & . & Millet commun \\
\hline Panicum miliaceum, épillet & imb. & . & . & . & . & . & 9 & . & . & Millet commun \\
\hline Triticum aestivum, rachis & carb. & 1 & . & . & . & . & . & . & . & Blé tendre \\
\hline Triticum, rachis & carb. & . & . & . & . & . & . & 3 & . & Blé tendre/dur \\
\hline Triticum dicoccon & carb. & . & . & . & . & . & . & 1 & . & Amidonnier \\
\hline Triticum spelta & & . & . & . & . & . & . & . & . & Epeautre \\
\hline Secale cereale, rachis & carb. & 49 & 45 & 454 & . & 6596 & 50 & 4770 & . & Seigle \\
\hline Secale cereale, rachis & imb. & 21 & . & . & 3 & . & 2632 & . & . & Seigle \\
\hline Setaria italica, épillet & carb. & . & . & . & . & . & . & . & . & Millet des oiseaux \\
\hline Setaria italica, épillet & imb. & . & . & . & . & . & 6 & . & . & Millet des oiseaux \\
\hline Setaria italica, glumelle & imb. & . & . & . & $\dot{.}$ & $\dot{.}$ & 3 & . & . & Millet des oiseaux \\
\hline Cerealia/Poaceae, noeuds & imb. & 30 & . & . & . & . & 6 & . & . & Céréales/Graminées \\
\hline Cerealia/Poaceae, noeuds & carb. & 12 & 20 & 79 & . & 339 & 12 & 43 & . & Céréales/Graminées \\
\hline Légumineuses & & & & & & & & & & \\
\hline Pisum sativum & carb. & 1 & 1 & . & . & 1 & . & . & . & Pois cultivé \\
\hline Vicia faba & carb. & . & 721 & $\dot{.}$ & $\dot{.}$ & 4 & 258 & $\dot{.}$ & $\dot{1}$ & Fèverole \\
\hline Vicia sativa agg. & carb. & . & 3 & . & $\dot{.}$ & . & . & . & 1 & Vesce cultivée \\
\hline Pisum/Vicia & carb. & $\dot{.}$ & . & $\dot{.}$ & $\dot{.}$ & $\dot{.}$ & $\dot{2}$ & $\dot{.}$ & . & Pois/Vesce \\
\hline & & & & & & & & & & \\
\hline Oléagineuses & & & & & & & & & & \\
\hline Linum usitatissimum & imb. & 27 & . & . & . & . & 337 & . & . & Lin cultivé \\
\hline Linum usitatissimum & carb. & . & $\dot{.}$ & $\dot{.}$ & $\dot{.}$ & 3 & 6 & $\dot{.}$ & $\dot{.}$ & Lin cultivé \\
\hline Linum usitatissimum, capsule & imb. & 3 & . & $\dot{.}$ & 2 & . & 236 & $\dot{.}$ & $\dot{.}$ & Lin cultivé \\
\hline & & & & & & & & & & \\
\hline Fruits & & & & & & & & & & \\
\hline Fruits secs/à coque & & & & & & & & & & \\
\hline Castanea sativa & imb. & 2 & . & . & 1 & . & 3 & . & . & Châtaignier \\
\hline Corylus avellana & imb. & 7 & . & . & 1 & . & 4 & . & 1 & Noisetier \\
\hline Corvlus avellana & carb. & . & . & . & . & 2 & . & 1 & . & Noisetier \\
\hline Fagus sylvatica, cupule & imb. & 5 & . & . & . & . & . & . & . & Hêtre \\
\hline Juglans regia & imb. & $10+1$ & . & . & . & . & 7 & . & 2 & Noyer commun \\
\hline Pinus pinea & imb. & 2 & . & . & . & . & . & . & . & Pin pignon \\
\hline Quercus, gland & carb. & . & . & 1 & . & . & . & . & . & Chêne \\
\hline Quercus, base gland & imb. & 7 & . & . & . & . & 16 & 1 & . & Chêne \\
\hline Quercus, coque & imb. & 3 & . & $\dot{.}$ & . & . & 1 & . & $\dot{.}$ & Chêne \\
\hline Quercus, cupule & carb. & . & . & 1 & . & . & . & . & . & Chêne \\
\hline & & & & & & & & & & \\
\hline Fruits à pépins & & & & & & & & & & \\
\hline Cydonia oblonga & imb. & 1 & . & . & . & . & . & . & . & Cognassier \\
\hline Ficus carica & imb. & 2 & . & . & 1 & . & . & . & . & Figuier \\
\hline Fragaria vesca & imb. & 103 & $\dot{.}$ & $\dot{.}$ & . & $\dot{.}$ & $\dot{9}$ & $\dot{.}$ & $\dot{.}$ & Fraisier des bois \\
\hline Malus, pépin & imb. & 20 & $\dot{.}$ & $\dot{.}$ & $\dot{.}$ & $\dot{.}$ & . & 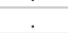 & $\dot{.}$ & Pommier \\
\hline Malus, fgt endocarpe & imb. & 4 & . & . & $\dot{.}$ & $\dot{.}$ & $\dot{.}$ & 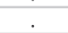 & . & Pommier \\
\hline Morus nigra & imb. & 2 & . & . & $\dot{.}$ & $\dot{.}$ & $\dot{.}$ & $\dot{.}$ & $\dot{.}$ & Mûrier noir \\
\hline Physalis alkekengi & imb. & 12 & . & . & $\dot{.}$ & $\dot{.}$ & $\dot{.}$ & $\dot{.}$ & $\dot{.}$ & Coqueret \\
\hline Pyrus, pépin & imb. & 219 & . & . & . & . & . & . & . & Poirier \\
\hline Pyrus, calice & imb. & 49 & $\dot{.}$ & $\dot{.}$ & $\dot{.}$ & $\dot{.}$ & $\dot{.}$ & $\dot{.}$ & $\dot{.}$ & Poirier \\
\hline Pyrus/Malus & imb. & 5 & . & $\dot{.}$ & $\dot{1}$ & . & $\dot{.}$ & $\dot{.}$ & . & Poirier/Pommier \\
\hline
\end{tabular}




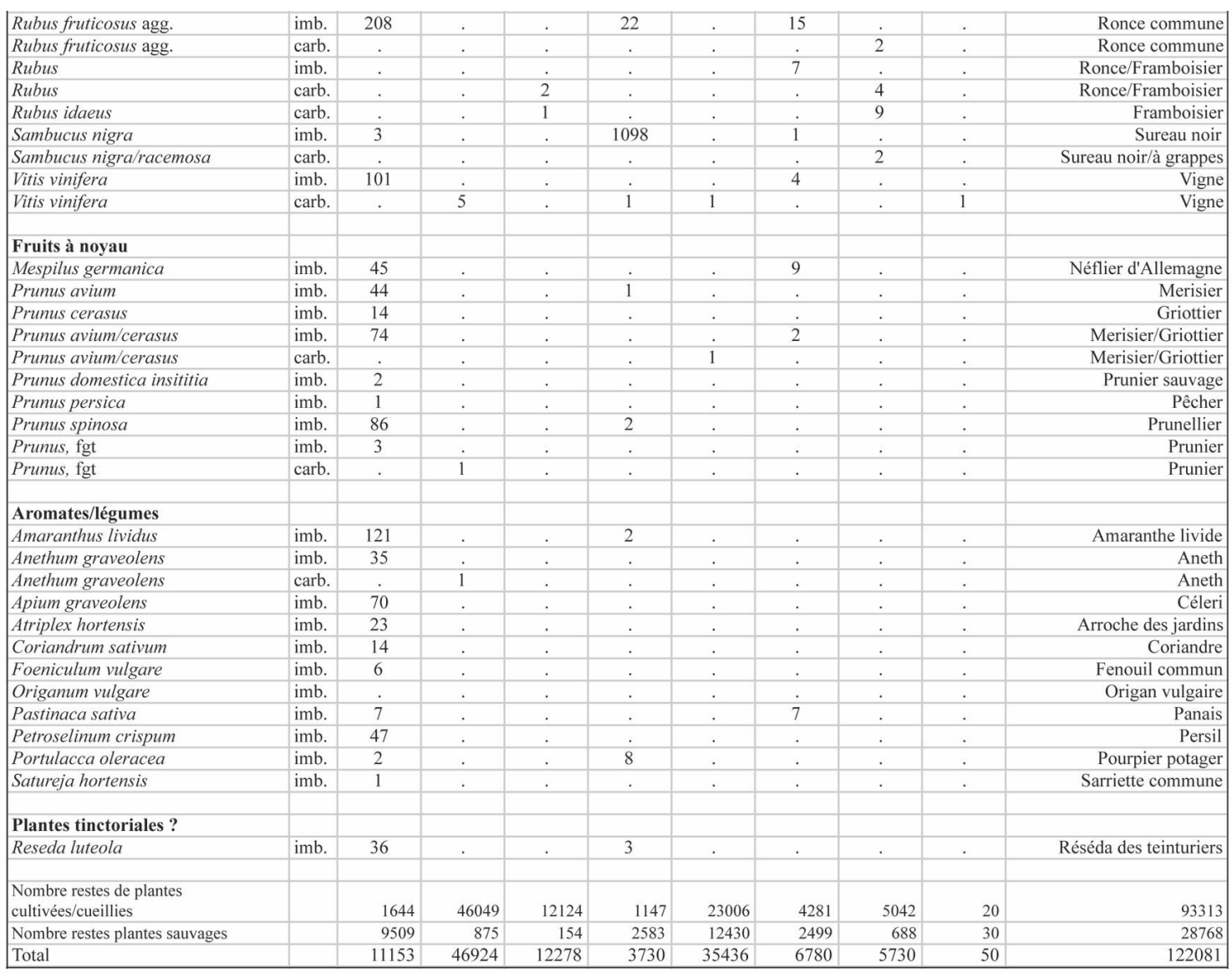

Abréviations : HtMA : haut Moyen Âge ; MAC : Moyen Âge central ; BMA : Bas Moyen Âge

$\mathrm{Hr}$ : habitat rural ; $\mathrm{V}:$ ville ; $\mathrm{BC}:$ basse cour ; $\mathrm{Ca}:$ Castrum ; ch : château

no : niveau d'occupation; $p$ : puits ; $f$ : fosse ; fé : fossé, sout : souterrain

c : carbonisé ; $\mathrm{i}$ : imbibé ; $\mathrm{m}$ : minéralisé ; cf : détermination taxonomique incertaine
Tableau 4 : Limousin : plantes cultivées et cueillies au Moyen Age.

Table 4: Limousin: cultivated and gathered plants during the Middle Ages.

été enregistrés. Ces espèces ne semblent pas être mentionnées en France au haut Moyen Âge, époque au cours de laquelle le lin prend une place croissante (Lepetz et al., 2002; Ruas, 2005).

Les fruitiers offrent un large corpus d'espèces provenant presqu'exclusivement du puits du Maupas. Les essences déterminées sont pour partie identiques à celles de l'époque gallo-romaine. De nouvelles sont cependant attestées, comme le merisier (Prunus avium), le cognassier (Cydonia oblonga), le néflier d'Allemagne (Mespilus germanica) et le mûrier noir (Morus nigra). Leur statut diffère en ce sens que la première est une espèce indigène, alors que les autres sont introduites. Le cognassier, qui serait originaire du Moyen Orient (Benzi et Berliocchi, 1999) ou du Sud-Ouest de l'Asie (Oberdorfer, 1994; Lauber et Wagner, 2000), demeure, de manière générale, discret dans les spectres fruitiers du Moyen Âge (Ruas et al., 2005-2006). Le néflier, dont l'origine est également incertaine, possède une répar- 
tition actuelle essentiellement atlantique ou subatlantique (Bonnier, 1992). Son introduction en Gaule non méditerranéenne date de l'époque romaine comme en témoignent des découvertes du i ${ }^{\text {er }}$ siècle en Bretagne (Ruas, 1992b). Enfin, le mûrier noir, espèce originaire du Caucase et du Nord de l'Iran (Benzi et Berliocchi, 1999), a également été introduit en Gaule à l'époque romaine. Il a été notamment identifié dans plusieurs latrines du nord de la France (Matterne, 2001). Parmi les autres espèces présentes figurent le châtaignier (Castanea sativa) (fig. 5) qui n’avait été déterminé qu’avec incertitude à l'époque précédente. Plusieurs espèces de tradition romaine apparaissent toujours dans le corpus des fruitiers : le figuier (Ficus carica), le pin pignon (Pinus pinea) et le pêcher (Prunus persica). Leur mise en évidence au haut Moyen Âge est rare aussi bien en France septentrionale que méridionale (Ruas et al., 2005-2006). Des pépins de figue ont été toutefois déterminés sur plusieurs sites du Baden-Württemberg (Allemagne) datés des $\mathrm{V}^{\mathrm{e}}$-VIII ${ }^{\mathrm{e}}$ siècles (Rösch, 1997, 1999, 2005, 2006b). Le large éventail d'espèces fruitières identifiées au Maupas ainsi que les quantités de restes pour certaines d'entre elles, permettent d'envisager le développement d'une arboriculture locale urbaine ou péri-urbaine dont les formes sont difficiles à définir : jardins fruitiers, vergers ou pré-vergers? Un commerce alimentaire avec le bassin méditerranéen n'est pas exclu.

Les aromates et les légumes sont représentés en grande quantité dans le puits du Maupas. Cette structure est la seule pour tout le Moyen Âge à avoir fourni un large spectre, les autres n'ayant livré que quelques restes et très peu de taxons. Les aromates identifiés, similaires à ceux mis en évidence à l'époque gallo-romaine, s'en différencient par l'absence d'épice et par la présence du persil (Petroselinum crispum). Le corpus des légumes se montre tout à fait différent de celui de la période précédente, avec l'amaranthe livide (Amaranthus lividus), l'arroche des jardins (Atriplex hortensis), le panais (Pastinaca sativa) et le pourpier potager (Portulacca oleracea). Aucun reste de calebasse n'a été identifié. Cette espèce n'est qu'exceptionnellement mentionnée au Moyen Âge (Ruas et al., 2005-2006).

\section{Le Moyen Âge central (XI'-XIII siècles)}

La fin de ce premier millénaire est marquée par des défrichements qui se font au détriment de la forêt et au bénéfice des champs et des landes. Celles-ci ont pu jouer un rôle important en constituant des zones de pacage et en fournissant la litière animale qui permettait une bonification des terres cultivées (Périgord, 1994). Ces espaces pouvaient être composés de genêt à balais (Cytisus scoparius), d'ajonc (Ulex), d'Éricacées (Ericaceae) et de fougère aigle (Pteridium aquilinum). Ces formations sont difficiles à mettre en évidence du point de vue palynologique car les grains de pollens de légumineuses sont très peu dispersés et rarement déterminables au niveau du genre ou de l'espèce (Allée et al., 1997).

Les sites archéologiques ayant fait l'objet d'une analyse carpologique sont localisés dans des contrées assez disparates. Ce sont les habitats castraux de la Renaudie à Masseret (Corrèze) et de Saint-Yrieix-la-Perche (Haute-Vienne) ainsi que l'habitat rural du Grandcher à Aix (Corrèze). Le premier est situé en pays d'Uzerche, à la limite du haut et du bas Limousin, le second dans la zone de transition entre le Limousin et le Périgord et le troisème, à proximité du Cantal, en Montagne limousine (fig. 1 et tableau 4).

\section{Productions et collectes végétales}

Les contextes archéologiques analysés sont constitués, pour le premier, de plusieurs fosses et de quelques trous de poteau, pour le second, d'un fossé, et pour le troisième, de trois fosses. Le nombre de restes pris en considération s'élève à 36277 inégalement répartis entre les trois sites. Deux modes de conservation sont attestés : la carbonisation et l'imbibition. Cette dernière n'a été mise en évidence qu'à Saint-Yrieix-la-Perche. Le spectre de ces trois occupations est donc assez différent. Deux d'entre eux ont livré de nombreux restes de céréales, le troisième, du fait d'une conservation imbibée à 99,7 \%, a essentiellement fourni des fruits.

Pour cette période deux concentrations de grains de céréales ont été mises en évidence, l'une à Aix, l'autre à Masseret. La première est constituée de près de 11000 grains d'avoine. Les bases d'épillet ou les épillets présents parmi les restes ont permis l'identification de l'avoine rude (Avena strigosa). En France, cette espèce bien adaptée aux sols secs, caillouteux et sableux, ne fait que rarement l'objet de déterminations. Elle est cependant connue en Bretagne où elle a été mise en évidence dans les Côtes-d'Armor et en Ille-etVilaine, sur les sites carolingiens du Camp de Péran (Ruas, 1992) et de Montours (Ruas et Pradat, 2001). Elle est plus fréquente aux Pays-Bas et en Allemagne du Nord où elle a été observée sur plusieurs sites, comme ceux de Gasselte (Van Zeist et Palfenier-Vegter, 1979), Pesse (Van Zeist et al., 1986), Middels (Behre, 1973), Schleswig (Pasternak, 1991) ou encore de Dalem et Flögeln-Dorf (Behre et Kučan, 1994). L'avoine rude est associée à plusieurs céréales, en particulier au seigle, ces deux espèces étant présentes dans les trois contextes. Les autres céréales identifiées sont le blé nu, l'orge vêtue et l'orge nue (Hordeum vulgare var. nudum) ${ }^{4}$ (fig. 5). La présence de cette dernière est particulière dans la mesure où celle-ci disparaît des corpus carpologiques au

4. Détermination confirmée par M. Rösch, U. Maier et E. Fischer. 
second âge du Fer (Matterne, 2001). Comme elle n'est attestée ici que par un seul grain, il est possible qu'elle ne soit que relictuelle et qu'elle ait eu davantage un statut de mauvaise herbe des champs que de céréale cultivée. La deuxième concentration est formée de grains de seigle associés à une proportion non négligeable de fragments de rachis et à des grains d'avoine. Plusieurs bases d'épillets ont permis, cette fois-ci, l'identification de l'avoine cultivée qui s'avère plus conforme aux données médiévales. Les autres céréales sont constituées par le blé nu, le millet commun, l'orge vêtue et le millet des oiseaux (Setaria italica). La présence de cette dernière est interéssante car peu fréquente au Moyen Âge (Ruas, 1998, 2005; Lepetz et al., 2002). L'amidonnier (Triticum dicoccon) a également été déterminé dans une autre structure dont l'assemblage carpologique est formé essentiellement de fragments de rachis de seigle.

Le statut de ces céréales peu fréquentes pose problème. S'agit-il de cultures locales, secondaires, d'adventices de culture ou de cultures fourragères? S'agit-il d'une diversification répondant à des besoins économiques, à l'exploitation de nouveaux terroirs ou encore à minimiser les risques de mauvaises récoltes? Si pour certaines d'entre elles, comme pour l'avoine rude, une culture locale peut être envisagée, il ne semble pas que cela puisse être possible pour l'amidonnier et le millet des oiseaux, identifiés par quelques exemplaires sur un seul site, ni pour l'orge nue dont un seul grain a été déterminé.

Les contextes analysés ne sont favorables ni aux légumineuses ni aux oléagineuses/textiles. Seule une graine de pois cultivé (Pisum sativum) et quatre de féverole (Vicia faba) ont été récoltées ainsi que trois semences et deux fragments de capsule de lin cultivé (Linum usitatissimum). Ces quelques éléments ne permettent pas de caractériser l'importance réelle des différentes légumineuses et oléagineuses.

Les structures de cette période n'ont pas livré beaucoup de fruitiers. La majorité des restes déterminés provient du fossé de la motte de Saint-Yrieix-la-Perche. Dix taxons dont neuf au rang d'espèces ont été identifiés. La mieux représentée en nombre d'éléments est le sureau noir (Sambucus nigra) avec près de 1100 semences. Les autres essences fruitières sont attestées de manière plus discrète : la ronce commune (Rubus fruticosus agg.), le prunellier (Prunus spinosa), le châtaignier (Castanea sativa), la vigne (Vitis vinifera), le néflier d'Allemagne (Mespilus germanica), le figuier (Ficus carica), le merisier (Prunus avium), le noisetier (Corylus avellana) et le pommier ou le poirier (Malus/Pyrus). Quelques fruitiers ont également été mis en évidence à Masseret : la vigne, le merisier ou cerisier et le noisetier. À Aix, trois taxons ont été identifiés : le chêne (Quercus), le framboisier (Rubus idaeus) et la ronce (Rubus).

\section{Le bas Moyen Âge (XIV-XVe siècles)}

À l'extrême fin du xve siècle, les données palynologiques indiquent une reconquête du milieu végétal (Tits-Dieuaide, 1994) qui se traduit par des défrichements touchant également les aulnaies des fonds de vallées des bas-plateaux jusque-là relativement préservées (Allée et Diot, 1997). L'augmentation de la population, associée à une exploitation extensive des milieux naturels, entraîne une recrudescence de l'érosion des sols perceptibles dans les analyses sédimentologiques (Bernard-Allée et Valadas, 1992; Valadas, 1982).

Seuls trois sites ont fait l'objet d'une analyse carpologique : l'habitat rural d'Aix-le Grandcher (Corrèze), la basse-cour de la motte féodale de Masseret-la Renaudie (Corrèze) et le castrum de Bas-Châlucet à Saint-Jean-Ligoure (HauteVienne). Les prélèvements sont issus de fosses, de niveaux d'occupation, d'un four, d'un foyer et d'un petit souterrain. Ce dernier est le seul contexte archéologique de cette période à avoir livré du matériel imbibé.

\section{Productions et collectes végétales}

Le nombre de restes déterminés pris en considération est de 9343, ce qui est inférieur aux deux phases précédentes du Moyen Âge. Leur répartition est inégale entre les sites, l'un d'eux, peu représentatif, n'ayant livré que vingt restes.

Les céréales mises en évidence sont les mêmes qu’à la phase précédente : avoines cultivée et rude (Avena sativa et $A$. strigosa), seigle (Secale cereale), orge vêtue (Hordeum vulgare), blé nu (Triticum aestivum/durum), millet commun (Panicum miliaceum) et millet des oiseaux (Setaria italica). L'avoine est mentionnée sur trois sites, le seigle, l'orge vêtue et le millet commun sur deux. L'avoine et le seigle sont les deux céréales à avoir livré le plus grand nombre de grains. Mais c'est surtout sous forme de rachis qu'est représenté le seigle. Ces éléments proviennent essentiellement de deux structures : le souterrain de Masseret et une unité d'habitation à Aix. Le millet commun a principalement été identifié sous forme de glumelles.

Deux légumineuses ont été déterminées : la féverole (Vicia faba) et la vesce cultivée (Vicia sativa agg.). La première a été mise en évidence à Masseret sous la forme d'un lot de 258 graines associées à plusieurs céréales et à du lin (fig. 6) et par un exemplaire unique à Saint-Jean-Ligoure. La seconde n'est mentionnée que sur ce dernier site. Si, pour l'ensemble du Moyen Âge, la féverole n'est attestée que sur quatre occupations, contre trois pour le pois cultivé et la vesce, en termes d'abondance, la première a livré deux concentrations importantes. Ces données concordent avec l'augmentation des occurrences observée au plan national (Lepetz et al., 2002; Ruas, 2005). La féverole a pu servir à l'alimentation humaine ou animale et 


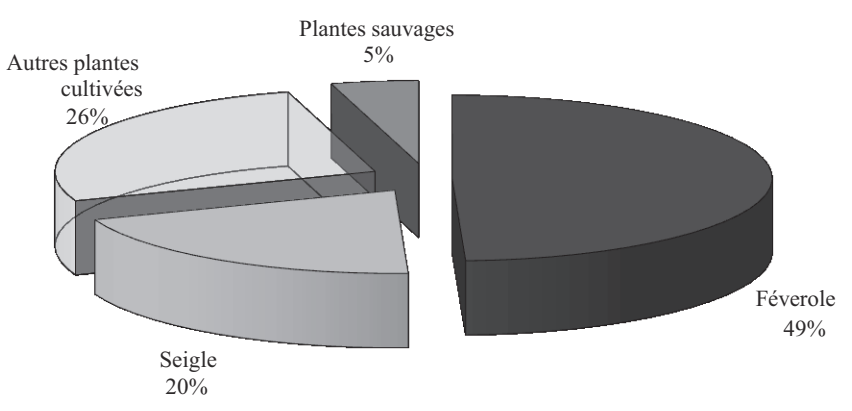

Figure 6 : Masseret (19), la Renaudie : Fréquence relative des plantes dans la fosse F 3152(nombre de restes : 470).

Figure 6: Masseret (19), la Renaudie : relative plants frequency in pit F 3152 (Number of plants remains: 470).

être exploitée en plein champ ou dans des jardins. Les sources écrites rendent compte également de la montée en puissance de cette légumineuse, en particulier pour le nord de la France (Le Roy Ladurie, 1994, 2004; Tits-Dieuaide, 1994; Vanderpooten, 2002). Les livres de recettes de la fin du Moyen Âge, bien que faisant référence à l'alimentation d'une certaine élite sociale, traduisent aussi ce goût porté aux légumineuses (Laurioux, 2002). Dans la mentalité médiévale, ces plantes sont considérées, à l'instar des céréales, comme des nourritures bien plus nobles que les légumes ou toute autre espèce croissant à même ou dans le sol (Grieco, 1993; Flandrin, 1993).

Parmi les oléagineuses/textiles, le lin cultivé (Linum usitatissimum) a été déterminé comme aux périodes précédentes. Il est associé à la moutarde noire, crucifère annuelle des champs, des friches et des décombres (Brugel et al., 2001). Aucune semence de chanvre n'a été encore découverte, mais la mise en évidence de pollens de chanvre ou de houblon (Cannabis/Humulus) dans plusieurs tourbières de la Creuse et de la Corrèze (Iskandar, 1992; Guenet, 1992; Valadas et Marambat, 1999; Dussot et Manville, 1990; Miras, 2004) laisse envisager un possible développement de sa culture aux époques historiques. Des semences de cette espèce ont été mises en évidence au second âge du Fer dans le Sud-Ouest de la France (Bouby, 2002).

Les fruitiers sont représentés par la vigne (Vitis vinifera), le néflier d'Allemagne (Mespilus germanica), le châtaignier (Castanea sativa) et le noyer (Juglans regia) ainsi que par plusieurs essences sauvages qui ont dû faire l'objet de collecte à des fins alimentaires : le noisetier (Corylus avellana), le chêne (Quercus), le fraisier des bois (Fragaria vesca), la ronce commune (Rubus fruticosus agg.) et le sureau noir (Sambucus nigra). Ils proviennent majoritairement du souterrain de Masseret et quelques-uns, d'Aix. Le mode de conservation intervient dans la composition du spectre car la plupart des restes issus du site castral sont imbibés. Il n'est donc pas possible de comparer le spectre des deux occupations pour discerner la part pouvant résulter de la fonction du site.

Un seul légume a été identifié, le panais (Pastinaca sativa), mentionné à Masseret sous forme imbibée. Cette espèce avait déjà été mise en évidence au haut Moyen Âge à Limoges le Maupas. Tout comme la carotte sauvage, cette ombellifère possède une racine qui a pu être récoltée à des fins alimentaires.

\section{ConClusion}

Basée sur la détermination de plus de 212000 restes imbibés, carbonisés et minéralisés et complétée par des données carpologiques publiées et inédites, cette synthèse apporte un éclairage nouveau sur les productions végétales et les collectes des végétaux aux époques protohistoriques et historiques dans l'ouest du Massif central.

Le corpus disponible pour la Tène finale, constitué de céréales, oléagineuses, fruitiers et aromates, est proche des données issues d'autres contrées françaises. Il convient de souligner l'importance qu'a pu prendre le millet commun à cette époque-là.

Les neuf études concernant la période gallo-romaine reflètent un spectre de plantes cultivées et cueillies beaucoup plus large. Les céréales vêtues jouent un rôle prépondérant, en particulier, l'orge vêtue, l'amidonnier, le millet commun et l'épeautre. Une survivance de l'engrain est à noter. La part du blé nu demeure difficile à évaluer. La fin de la période voit l'introduction du seigle. Les légumineuses sont représentées par plusieurs espèces comme la lentille cultivée, le pois cultivé, la féverole et la vesce cultivée, ainsi que les oléagineuses/textiles avec l'attestation de lin, de pavot et de caméline. La gamme des essences fruitières est très ouverte, marquée par des influences méditerranéennes. Plusieurs espèces ont pu faire l'objet d'importations ou d'une acclimatation comme le figuier, le pin pignon, le pêcher, le griottier, la vigne, etc. C'est le cas aussi des aromates et des légumes. Parmi les épices, la mise en évidence de graines de poivre au haut-Empire est à souligner du fait du caractère exceptionnel de cette découverte. Cette épice, originaire du continent indien, est connue par les sources écrites pour avoir été une denrée particulièrement appréciée et recherchée des Romains.

Pour le Moyen Âge, le corpus des données provient de huit occupations couvrant les différentes séquences de cette période. On note une modification du spectre des céréales avec le déclin prononcé de l'épeautre et de l'amidonnier et un essor important du seigle, de l'avoine et du blé nu (fig. 7 et 8). Épeautre et amidonnier ne font l'objet que de quelques déterminations isolées. Cependant, les mentions de 


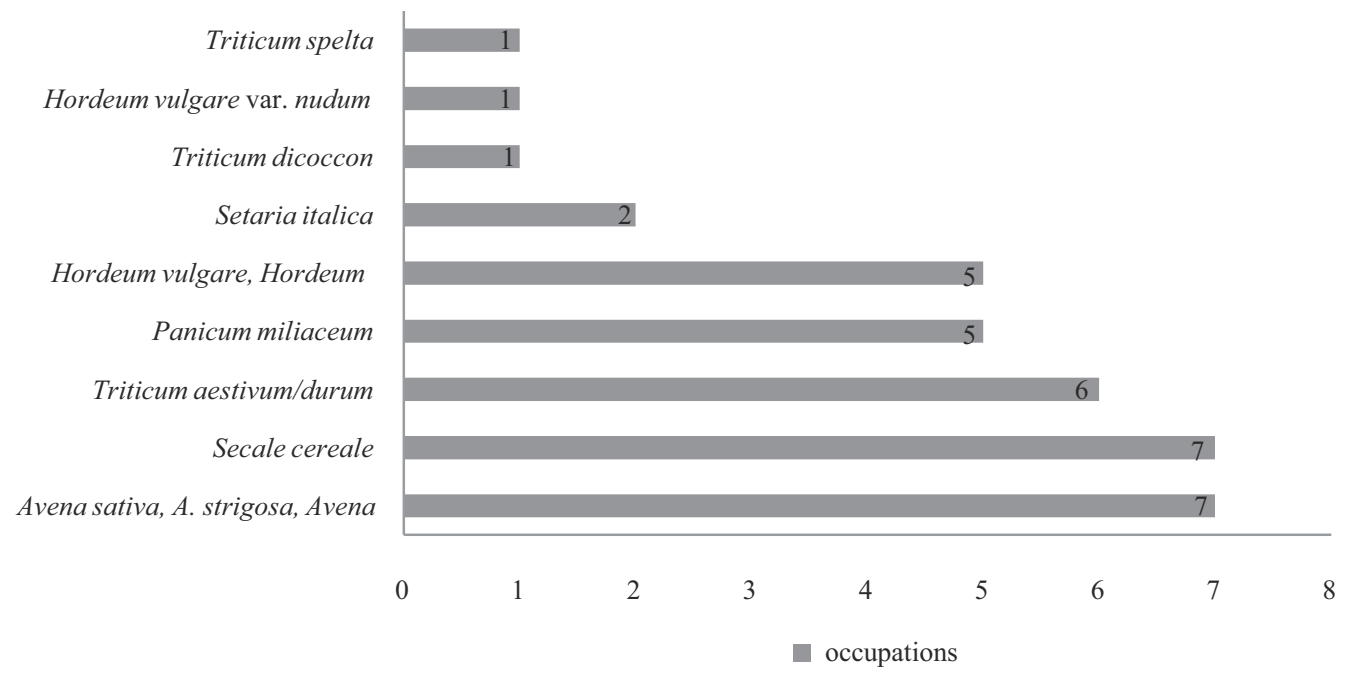

Figure 7 : Occurrence des céréales par occupation pour le Moyen Âge (Nb : 8).

Figure 7: Occurrence of cereals per occupation during the Middle Ages (Nb: 8).

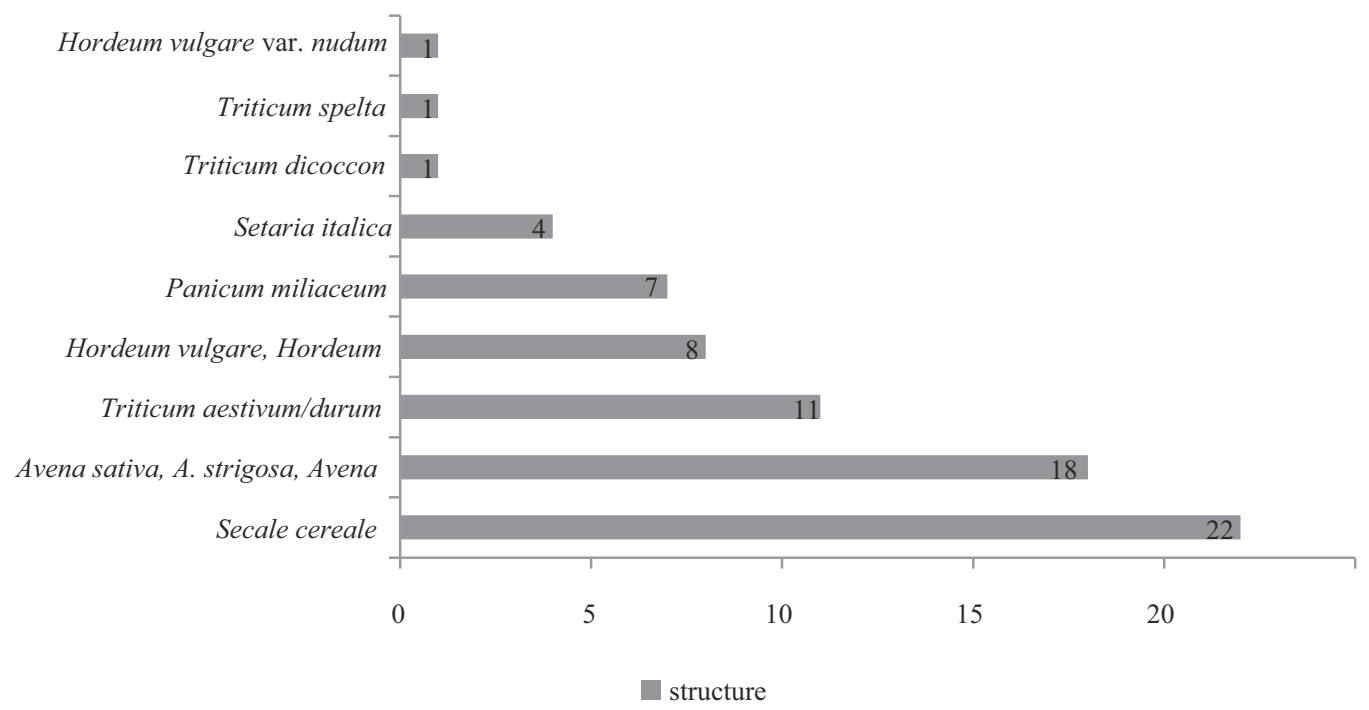

Figure 8 : Occurrence des céréales par structure pour le Moyen Âge (Nb : 22).

Figure 8: Occurrence of cereals per structure during the Middle Age (Nb: 22).

ces plantes demeurent peu fréquentes pour le Moyen Âge en France. Il en va de même pour le millet des oiseaux, identifié sur deux phases d'occupation d'un même site et pour l'avoine rude, espèce rarement mise en évidence en France à l'exception de quelques sites de l'Ouest. Les spectres des légumineuses et des oléagineuses/textiles se montrent moins diversifiés qu'à l'époque gallo-romaine. La féverole semble occuper une place importante de même que le lin cultivé. Le corpus des fruitiers reste très diversifié. Apparaissent quelques nouvelles espèces, comme le mûrier noir, le néflier d'Allemagne et, de manière plus inattendue, le cognassier.
Au contraire, le spectre des aromates s'apauvrit, ceux-ci n'étant attestés qu’au haut Moyen. Aucune épice n'a été mise en évidence. Le corpus des légumes enregistre une modification des espèces déterminées. Toutefois, ces plantes sont rarement mises en évidence sur les sites analysés.

De nouvelles études en cours vont permettre de compléter notre connaissance sur la production et la collecte des végétaux et la gestion des ressources naturelles en Limousin aux périodes proto-historiques et historiques. 


\section{Remerciements}

Nous tenons à remercier tout particulièrement les responsables d'opération, ceux de l'INRAP (ex-AFAN), Christine Best, Christophe Maniquet, Patrick Massan, et ceux du Service Régional de l'Archéologie du Limousin, Patrice Conte et Guy Lintz, qui nous ont confié l'analyse de prélèvements de plusieurs sites. Tous nos remerciements vont également à MarieFrançoise Diot, du Centre National de Préhistoire de Périgueux pour son aide, ainsi qu'à Helmut Kroll, Responsable de l'Institut für Ur-und-Frühgeschichte de l'Université Christian Albrecht de Kiel (Allemagne), aux professeurs Bernard Valadas ( $\dagger$ ), de la Faculté des Lettres et des Sciences Humaines de Limoges, Michel Botineau, de la Faculté de Médecine et de Pharmacie de Limoges et Thierry Gauquelin, de l'université Paul-Sabatier (Toulouse 3), pour leurs remarques avisées et leurs conseils. Nous tenons à remercier également Helmut Schlichterle, Directeur de la Pfahlbauarchäologie Bodensee-Oberschwaben du Landesdenkmalamt Baden-Württemberg et toute son équipe pour son accueil et son aide matérielle ayant rendu possible un grand nombre de ces analyses archéobotaniques. Nous remercions également les relecteurs, en particulier M.-P. Ruas ainsi que la personne anonyme, pour leurs remarques avisées.

\section{Bibliographie}

Allée, P. (dir.), Ballet, I., Denis, J., Diot, M.-F., DurieuX, J.-F., Gebhart, A., Petit, F., Valadas, B., 1996. Archéologie agraire en Limousin, Projet Collectif de Recherche 1994-1996, Rapport, Service Régional de l'Archéologie du Limousin, Université de Limoges, CNRS-Upresa 6042, Clermont-Ferrand.

Allée, P., Diot, M.-F., 1997. PCR " Archéologie agraire en Limousin ", In Bilan Scientifique 1996, éd. Direction Régionale des Affaires Culturelles du Limousin, p. 56-57.

Allée, P., Diot, M.-F., Durieux, J.-F, Reynet, J.-M., Valadas, B., 1997. Trois mille ans d'enregistrement sédimentaire dans les fonds de vallons des bas plateaux limousins, in La dynamique des paysages protohistoriques, antiques, médiévaux et modernes. XVII ${ }^{\mathrm{e}}$ Rencontres Internationales d'Archéologie et d'Histoire d'Antibes, éd. APDCA, Sophia Antipolis, p. 365-387.

André, J., 1961. L'alimentation et la cuisine à Rome, Paris, Klincksieck.

BAKELS, C., 1984. Carbonized seeds from Northern France, Analecta Praehistorica Leidensia, 17, p. 1-27.

BaKels, C., 1991. Western Continental Europe, in Van Zeist W., Wasilykowa K., Behre K.-E. (dir.), Progress in Old World Paleoethnobotany. An retrospective view on the occasion of 20 years of the International Work Group for Palaeoethnobotany, A. A. Balkema, Rotterdam, Brookfield, p. 279-298.
BAKELS, C., 1999. Archaeobotanical investigations in the Aisne valley, northern France, from the Neolithic up to the early Middle Ages. Vegetation History and Archaeobotany, p. 71-77.

BaKels, C., 2005. Crops produced in the southern Netherlands and northern France during the early medieval period : a comparison. Vegetation History and Archaeobotany, 14, p. 394-399.

BARBIER, D., 1999. Histoire de la végétation du nord-mayennais de la fin du Weisélien à l'aube du Xxi siècle. Mise en évidence d'un Tardiglaciaire armoricain. Interaction hommemilieu, Thèse de doctorat. Université de Nantes, Laboratoire d'Ecologie et des paléoenvironnements atlantiques, France, éd. Groupe d'étude des milieux naturels, Nantes.

BEHre, K.-E., 1973. Mittelalterliche Kulturpflanzenfunde aus der Kirche von Middels (Stadt Aurich/Ostfriesland), Probleme der Küstenforschung im südlichen Nordseegebiet, 10, Verlagsbuchhandlung August Lax, Hildesheim, p. 39-47.

Behre, K.-H, Kucan, D., 1994. Die Geschichte der Kulturlandschaft und des Ackerbaus in der Siedlungskammer Flögeln, Niedersachsen, seit der Jungsteinzeit. Probleme der Küstenforschung im südlichen Nordseegebiet, Illsensee Verlag, Oldenburg.

Benzi, F., Berliocchi, L., 1999. L'histoire des plantes en Méditerranée. Art et botanique, éd. Actes Sud/Motta, Arles.

Bernard-Allée P., Valadas, B., 1992. L'état des recherches sur l'archéologie agraire en Limousin. Norois, 39, (156), p. 509518.

Bernard-Allée, P., Valadas, B. 1993. Archéologie agraire en Limousin : l'apport des remblaiements des fonds de vallons et des banquettes de cultures, Travaux d'Archéologie Limousine, 13, p. 7-18.

Bernard-Allée, P., André, M.-F., Pallier, G., Desbordes, O. (dir), 1994. Atlas du Limousin, Limoges, Presses Universitaires de Limoges.

Bonnier, G., rééd. 1992. La grande flore en couleur de G. Bonnier, Paris, Belin, 4 tomes.

Bouby, L., 1998. Two early finds of gold-of-pleasure (Camelina sp.) in middle Neolithic and Chalcolithic sites in western France, Antiquity, 72, p. 391-398.

Bouby, L., 2002. Le chanvre (Cannabis sativa L.) : une plante cultivée à la fin de l'âge du fer en France du Sud-Ouest?, C.R. Palevol., 1, p. 89-95.

Bouby, L., LÉA, V., 2006. Exploitation de la vesce commune (Vicia sativa) au Néolithique moyen dans le Sud de la France. Données carpologiques du site de Claparousse (Lagnes, Vaucluse), C.R. Palevol, 5, p. 973-980.

Bouby, L., Marinval, P., 2004. Fruits and seeds from Roman cremations in Limagne (Massif central) and the spatial variability of plant offerings in France, Journal of Archaeological Science, 31, p. 77-86. 
Bouchette, A., 2004. Le temps des récoltes. Agriculture et alimentation végétale du Néolithique à la Renaissance en Limousin, Thèse de Doctorat, Université Paul Sabatier, Toulouse III, UFR Sciences de la Vie et de la Terre.

Bournerias, M., 1979. Guide des groupements végétaux de la région parisienne, éd. Masson, Paris.

Brombacher, C., 1997. Archaeobotanical investigations of Late Neolithic lakeshore settlements (Lake Biel, Switzerland), Vegetation History and Archaeobotany, 6, p. 167-186.

Brugel, E., Brunerye, L., Vilks, A. (dir.), 2001. Plantes et végétation en Limousin. Atlas de la flore vasculaire, éd. Espaces Naturels du Limousin. Conservatoire Régional des Espaces Naturels du Limousin, Saint-Gence.

Burnouf, J., Chouquer, G., Bravard, J.-P. (dir.), 1997. La dynamique des paysages protohistoriques, antiques, médiévaux et modernes. In : Rencontres internationales d'Archéologie et d'Histoire d'Antibes. Actes des Rencontres, 19-20-21 octobre 1996, éd. CNRS.

Carruthers, W. J., 1991. Mineralised Plant Remains : Some Examples from Sites in Southern England. In E. Hajnalova (Hrsg.) Palaeoethnobotany and Archaeology. International Work-Group for Palaeoethnobotany $8^{\text {th }}$ Symposium Nitra-Nové Vozokany 1989. ACTA 7. Nitra, p. 75-80.

Colombain, J.-P., Lombard, R., 1991. L'habitat rural d'Esplaux (Commune de Sarran, Corrèze), Travaux d'Archéologie Limousine, p. 113-128.

Comet, G., 1992. Le Paysan et son outil. Essai d'histoire technique des céréales (France, VIII $-X V^{e}$ siècle), éd. École Française de Rome, Palais Farnèse, Rome.

Conedera, M., Krebs, P., Tinner, W., Pradella, M., Torriani, D., 2004. The cultivation of Castanea sativa (Mill.) in Europe, from its origin to its diffusion on a continental scale. Vegetation History and Archaeobotany, 13, p. 161-179.

Couderc, J.-M., Provost, M., 1990. Vigne et vignoble ligériens dans l'Antiquité, Archéologie de la vigne et du vin. Caesarodunum, 24, p. 89-98.

Cour, P., Duzer, D., Planchais, N., 1973. Analyses polliniques de l'atmosphère de Montpellier. Document correspondant à la phénologie de la floraison de la vigne en 1972, Naturalia monspeliensia, ser. Bot., 23-24, p. 225-229.

Cyprien, A., L., Visset, L., Carcaud, N., 2004. Evolution of vegetation landscapes during the Holocene in the central Loire basin and downstream (Western France), Vegetation History and Archaeobotany, 13, (3), p. 181-196.

De Hingh, A., 1993. Bottle gourd seeds at Gallo-Roman "Le Bois Harlé" (Oise, France), Analecta Praehistorica Leidensia, 26, p. 93-97.

Denèfle, M., Valadas, B., Vilks, A. et Lintz, G., 1980. L'évolution holocène de la végétation en Limousin, Bulletin de l'Association Française d'Étude du Quaternaire, 4, p. 189-198.
Desbordes, J.-M., Valadas, B., 1998. Aux origines du parcellaire en Montagne limousine : l'exemple d'une banquette agricole à Maurianges (commune de Chaumeil, Corrèze), Travaux d'Archéologie Limousine, 18, p. 99-104.

Desprat, S., Sanchez Goni, M. F., Loutre, M. F., 2003. Revealing climatic variability of the last three millenia in northwestern Iberia using pollen influx data, Earth and Planetary Science Letters, 213, p. 63-78.

Diétrich, A., Ruas, M.-P., 1990. Des hommes et des plantes au $\mathrm{II}^{\mathrm{e}}$ siècle à Mazières-en-Mauges, In Mazières-en-Mauges (Maineet-Loire) de la préhistoire à l'époque romaine, éd. Association mazièraise d'Archéologie, p. 27-32.

Diot, M.-F., 2002. Étude palynologique des dolmens de Bois Neuf III, à Marsac (Creuse) et Bagnol à Fromental (HauteVienne), Bulletin de la Société Préhistorique Française, 99, (1), p. 91-103.

Diot, M.-F., Miras, Y., Pontet, A., 2006. Analyse pollinique de la tourbière de l'ancien étang de la villa gallo-romaine des Cars (Corrèze). Environnement et peuplement de la moyenne montagne du Tardiglaciaire à nos jours, in Y. Miras, F. Summerly (dir.), Actes de la Table-Ronde de Pierrefort, Besançon, Presses Universitaires de Franche-Comté, (Annales Littéraires, 799 Série Environnement, Société et Archéologie), p. 109-121.

Dubois, G., Dubois, C., 1944. Remarques sur le Quaternaire et les tourbières de la Montagne limousine de Millevaches, Bulletin de la Société de Géologie de France, 5, (14), p. 29-36.

Dubois, G., Dubois, C., Glangeaud, L., 1942. Sur le Quaternaire du plateau de Millevaches et le profil pollinique d'une tourbière à Barsangues, Revue des Sciences naturelles d'Auvergne, 8, p. 164-177.

Dussot, D., Lintz, G., Vuaillat, D., 1992. La sépulture gauloise de Boiroux, commune de Saint-Augustin (Corrèze), Aquitania, 10 , p. 5-26.

Dussot, D., Manville, M. 1990. Occupation du sol et dynamique des paysages : le vallon des bains (commune de SainteFeyre, Creuse), Travaux d'Archéologie Limousine, 11, p. 7-32.

Dussot, D., Roger, J., Beausoleil, J.-M., 2002. La sépulture gallo-romaine de Fontvieille (Vareilles, Creuse), Aquitania, 18, p. $157-170$.

Flandrin, J.-L., 1993. Les légumes dans les livres de cuisine français, du XIV au XVIII ${ }^{\mathrm{e}}$ siècle, In A. J. Grieco, O. Redon, L. Tongiorgi Tomasi (dir.), Le monde végétal (XII -XVIt siècles), Saint-Denis, Presses Universitaires de Vincennes, coll. « Essais et Savoirs ", p. 71-85.

Fredon, J.-J., 1985. Macrorestes végétaux de sites archéologiques en Limousin, Travaux d'Archéologie Limousine, 1984, 5 : 7-15.

Fredon, J.-J., 1995. Contribution de l'anthracologie à la connaissance de l'évolution du couvert végétal en Limousin, Travaux d'Archéologie Limousine, 1994, 15, p. 19-29.

Freytet, P., Valadas, B., Blanchard-Lemée, M., Denèfle, M., Dussot, D., Duperron, J., 1989. Le site de la Védrenne 
(La Chapelle Saint-Martial, Creuse). Étude d'une villa galloromaine et de son environnement, Travaux d'Archéologie Limousine, 10, p. 33-43.

Göldner, H., KreuZ, A., 1999. Begraben an einem „stillen Ort“. Botanische und archäologische Funde als neue Hinweise zum Leben im römischen Dieburg, Denkmalpflege und Kulturgeschichte, 2, p. 10-17.

Green, F. J., 1979. Collection and interpretation of botanical information from medieval urban excavations in Southern England, Archaeo-Physika, 8, p. 39-55.

GreIG, J., 1982. Garderobes, sewers, cesspits and latrines. Current Archaeology, 85, p. 49-52.

Grieco, A. J., 1993. Les plantes, les régimes végétariens et la mélancolie à la fin du Moyen Âge et au début de la Renaissance italienne, in J. Grieco Allen, O. Redon, L. Tongiorgi Tomasi, Le monde vegetal (XII-XVIT siècle) savoirs et usages sociaux, SaintDenis, Presses Universitaires de Vincennes, p. 11-29.

GuENET, P., 1992. L'impact de l'homme sur son environnement en moyenne montagne, du Cézallier au plateau de Millevaches (Massif central, France), $117^{e}$ Congrès national des sociétés savantes, Moyenne montagne, p. 515-528.

Guenet, P., 1993. Analyses pollenanalytiques en Artense et sur le plateau de Millevaches (Massif central, France), Palynosciences, 2, p. 79-107.

HeIM, J., 1970. Les relations entre les spectres polliniques récents et la vegetation actuelle en Europe occidentale, Thèse de l'Université de Louvain, Belgique.

Hellwig, M., 1997. Plants remains from two cesspits $\left(15^{\text {th }}\right.$ and $16^{\text {th }}$ century) and a pond $\left(13^{\text {th }}\right)$ from Göttingen, southern Lower Saxony, Germany. Vegetation History and Archaeobotany, 6, p. 105-116.

Hopf, M., 1991. South and Southwest Europe, Progress in Old World Palaeoethnobotany, éd. Balkema, Rotterdam, p. 241277.

IsKANDAR, D., 1992. Analyse palynologique de la tourbière de Montmary à Saint-Maixant (Creuse). Travaux d'Archéologie Limousine, 12, p. 155-161.

JACOMEт, S., 1988. Pflanzen mediterraner Herkunft in neolithischen Seeufersiedlungen der Schweiz. Der prähistorische Mensch und seine Umwelt, Festschrift für U. Körber-Grohne, p. 205-212.

Jacomet, S., Kreuz, A., 1999. Archäobotanik, Verlag Eugen Ulmer, coll. UTB für Wissenschaft, Stuttgart.

JACOMET, S., Schibler, J., 2002. Die archäobiologischen Untersuchungen auf den Ausgrabungen 1999 und 2000 in Biesheim/Kunheim. In H. U. Nuber, M. Reddé, Das römische Oedenburg (Biesheim/Kunheim, Haut-Rhin, France). Frühe Militärlager, Strassensiedlung und valentinianische Festung. Le site romain d'Oedenburg (Biesheim/Kunheim, Haut-Rhin, France). Les camps militaires précoces, le vicus et la forteresse de Valentinien. Germania, 80, (1), p. 169-242.
JALUT, G., 1984. L'action de l'homme sur la forêt montagnarde des Pyrénées ariègeoises et orientales depuis 4000 B.P. d'après l'analyse pollinique. Actes $106^{e}$ Congrès national des Sociétés Savantes, Perpignan CTHS Sect. Géographie (1981), p. 163172.

Jalut, G., Delibrias, G., Dagnac, J., Mardones, M. Bouhours, M., 1982. A palaeoecological approach to the last 21000 years in the Pyrenees : the peat bog of Freychinède (alt. $1350 \mathrm{~m}$, Ariège, South France). Palaeogeography, Palaeoclimatology, Palaeoecology, 40, p. 321-359.

KARG, S., 1996. Einmal gegessen und verdaut - zum Nachweis von Nahrungsmittelpflanzen in Latrinenablagerungen, in $\mathrm{P}$. Kamber, C. Keller (dir). Katalog der Austellung Fundgruben - Stille Örtchen ausgeschöpft, Historisches Museum, Basel, p. 69-74.

Kenla, J.-V., Jalut, G., 1979. Déterminisme anthropique du développement du hêtre dans la sapinière du Couserans (Pyrénées ariégeoises, France) durant le Subatlantique. Géobios, 12, (5), p. 735-738.

KNÖRZER, K. H., 1984. Aussagemöglichkeiten von paläoethnobotanischen Latrinenuntersuchungen, in W. Van Zeist et W. A. Casparie (dir.), Plants and Ancient Man. Studies in palaeoethnobotany, $6^{\text {th }}$ Symposium Palaeoethnobotany, Groningen, p. 331-338.

KreUZ, A., 1994/1995. Landwirtschaft und ihre ökologischen Grundlagen in den Jahrhunderten um Christi Geburt : zum Stand der naturwissenschaftlichen Untersuchungen in Hessen. Berichte der Kommission für Archäologische Landesforschung in Hessen, 3, p. 59-91.

KuČAN, D., 1984. Der erste römerzeitliche Pfefferfund - nachgewiesen im Legionslager Oberaden (Stadt Bergkamen), Ausgrabungen und Funde in Westfalen-Lippe, 2, p. 51-56.

KuČAn, D., 1992. Die Pflanzenreste aus dem römischen Militärlager Oberaden, Bodenaltertümer Westfalens 27. Das Römerlager in Oberaden III, p. 237-265.

Lauber, K., Wagner, G., 2000. Flora Helvetica. Flore illustrée de Suisse, Paris, Belin.

Laurioux, B., 2002. Manger au Moyen Agge. Pratique et discours alimentaires en Europe aux XIV et $X V^{e}$ siècles, Paris, Hachette, coll. «Littérature".

Lavergne, D., 1963. Carte de la végétation de la France, $\mathrm{n}^{\circ} 57$, Bergerac, éd CNRS, Toulouse, 1/250000.

Lavergne, D., 1969. Carte de la végétation de la France, $\mathrm{n}^{\circ} 51$, Limoges, éd CNRS, Toulouse, 1/250000.

LeFÈVRe, F., 1990. Vin et viticulture en pays rème à l'époque romaine, in Archéologie de la vigne et du vin, Caesarodunum, 24, p. 163-174.

LeméE, G., 1943. L'évolution forestière post-glaciaire du Limousin méridional d'après l'analyse pollinique, Compte-rendus de l'Académie des Sciences, Paris, série D, 216, p. 74-75.

ArcheoSciences, revue d'archéométrie, 35, 2011, p. 65-92 
LEMÉE, G., 1949. Synchronisation entre l'Âge du Bronze et les phases forestières du plateau de Millevaches, Bulletin de la Société Préhistorique Française, 66, p. 68-71.

LEMÉE, G., 1952. Le cadre végétal au début de la période galloromaine sur le plateau de Millevaches, Bulletin de la Société Préhistorique Française, 69, p. 195-196.

LEMÉE, G., 1980. Évolution postglaciaire et récente de la végétation des Monts de la Marche d'après l'analyse pollinique, Bulletin de la Société botanique de France, 127, p. 59-69.

Lepetz, S., Matterne, V., Ruas, M.-P., Yvinec, J.-H., 2002. L'agriculture et l'élevage en France septentrionale de l'âge du Fer à l'An Mil. Approches carpologique et archéozoologique, in A. Belmont (dir.), Autour d'Olivier de Serres. Pratiques agricoles et pensée agronomique du Néolithique aux enjeux actuels, Actes du colloque, Le Pradel, Ardèche, 27-29 septembre 2000. Association d'Histoire des Sociétés Rurales. Bibliothèque d'Histoire Rurale, 6, p. 70-108.

Le Roy Ladurie, E. (dir.), 1994. Paysages, paysans. L'art et la terre en Europe du Moyen Âge au XX siècle, éd. Bibliothèque Nationale de France, Réunion des Musées Nationaux, Paris.

Le Roy Ladurie, E., 2004. Histoire humaine et comparée du climat. Vol.1. Canicules et glaciers XIII s.-XVIIt s., Paris, Fayard.

LinTZ, G., 2000. Saint-Gence, Le Patureau. Bilan scientifique de la région Limousin 1999, DRAC Limousin, SRA, éd. Ministère de la Culture et de la Communication, Paris, p. 44-46.

Lorquin, A., Moulherat, C., 2001-2002. Étude des vestiges textiles de la sépulture gallo-romaine de Fontvielle à Vareilles (Creuse), Aquitania, 18, p. 171-172.

Magny, M., Begeot, C., Guiot, J., Peyron, O., 2003. Contrasting patterns of hydrological changes in Europe in response to Holocene climate cooling phases, Quaternary Science Reviews, 22, p. 1589-1596.

Malrain, F., Matterne, V., Méniel, P., 2002. Les paysans gaulois (III siècle-52 av. J.-C.), éd. Errance/INRAP, Paris.

Mane, P., 1994. Le paysan mis en scène, in E. Le Roy Ladurie (dir.), Paysages, paysans. L'art et la terre en Europe du Moyen Âge au $X X^{e}$ siècle, éd. Bibliothèque Nationale de France, Réunion des Musées Nationaux, p. 43-47.

Maniquet, C. (dir.), 1999. Chantier de la Faculté de Droit (2e phase). Le "sanctuaire augustéen" d'Augustoritum et l'évolution d'un îlot situé au pied du forum, DFS, SRA Limousin, AFAN, Rectorat de l'Académie de Limoges, 2 tomes.

Marguerie, D., 1992. Évolution de la végétation sous l'impact humain en Armorique du Néolithique aux périodes historiques, Travaux du Laboratoire d'Anthropologie de Rennes, Université de Rennes 1, UPR n ${ }^{\circ} 403$ du CNRS.

Marinval, P., 1986. Bilan des analyses paléocarpologiques du Mésolithique jusqu'à l'époque médiévale en CentreOuest. Préhistoire du Poitou-Charentes, problèmes actuels, Actes du $111^{\mathrm{e}}$ Congrès national des Sociétés Savantes, Pré- et Protohistoire, Poitiers, p. 243-253.
Marinval, P., 1988. Cueillette, agriculture et alimentation végétale de l'Epipaléolithique jusqu'au second Age du Fer en France méridionale. Apport palethnographiques de la carpologie. Thèse de Doctorat (Nouveau Régime), École des Hautes Études en Sciences Sociales, Paris, 2 volumes dactylographiés.

Marinval, P., 1991. Analyse carpologique de deux incinérations aux Ribières, Bessines-sur-Gartempe (Haute-Vienne), Travaux d'Archéologie Limousine, 1, p. 90-93.

Marinval, P., 1993. Étude carpologique d'offrandes alimentaires végétales dans les sépultures gallo-romaines : réflexions préliminaires. Monde des morts, monde des vivants en Gaule rurale ( $e^{e}$ s. av. J.-C.-Ve s. apr. J.-C.), Actes du Colloque Archéa/Ager, Orléans, Conseil Régional, 7-9 février 1992, Tours, p. 45-65.

Marinval, P., 2001. Offrandes végétales de la nécropole galloromaine des Sagnes à Pontarion (Creuse), in G. Lintz (dir.), La nécropole gallo-romaine des Sagnes à Pontarion (Creuse), éd. Association des Publications Chauvinoises, Chauvigny, Mémoire 20, p. 191-193.

Marinval, P., 2002. Permanences et innovations agricoles en France méridionale du Néolithique à l'Antiquité romaine, in A. Belmont (ed), Autour d'Olivier de Serres. Pratiques agricoles et pensée agronomique du Néolithique aux enjeux actuels, Actes du colloque, Le Pradel, Ardèche, 27-29 septembre 2000. Association d'Histoire des Sociétés Rurales, Bibliothèque d'Histoire Rurale, 6, p. 11-19.

Marinval, P., 2005. Plantes à huile en France du mésolithique à l'Antiquité, in F. Sigaut, M. Marinval, M. Gast, Plantes et moulins à huile, hier et demain, Archéo-Plantes. Hommes et Plantes de la Préhistoire à nos jours, éd. AITAE, Centre d'Anthropologie, AEP, 1, p. 13-39.

Marinval, P., Maréchal D., Labadie D., 2002. Arbres fruitiers et cultures jardinées gallo-romains à Longueil-Sainte-Marie (Oise), Gallia, 59, p. 253-271.

Massan, P., Petit, F., Ernaux, P., Pironnet, C., Dalle, S., Vequaud, B., Fondeville C., avec la coll. de Stutz, F., Denis, J., Diot, M.-F., Wattez, J., Milor, F., Dussot, D., Bouchette, A., Galibert, P., 2002. La Gare routière de la rue Charles-Gide, Limoges, Document final de synthèse, AFAN, SRA Limousin.

Matterne, V., 2001. Agriculture et alimentation végétale durant l'âge du Fer et l'époque gallo-romaine en France septentrionale, Montagnac : Monique Mergoil, Archéologie des plantes et des animaux, 1, $312 \mathrm{p}$.

Matterne, V., 2003. Nouvelles données carpologiques relatives à la période gallo-romaine en régions Picardie et Ile-de-France, Besançon. Actualité de la recherche en Histoire et Archéologie Agraires, Actes du colloque international AGER V, septembre 2000, Presses Universitaires Franc-Comtoises, Annales Littéraires, Série "Environnement, Sociétés et archéologie ", 5, p. 241-268. 
MeIFFren, I., 1984. Étude du contenu pollinique de l'atmosphère de la région toulousaine (Résultats de trois années d'observation), Thèse de Doctorat, Université Paul Sabatier de Toulouse, France.

Miras, Y., 2001. Pour une histoire de la végétation sur le plateau de Millevaches : méthodologie, concepts et premiers résultats, Travaux d'Archéologie Limousine, 21, p. 7-15.

Miras, Y., 2004. L'analyse pollinique du plateau de Millevaches (Massif central, France) et de sites périphériques limousins et auvergnats : approche des paléoenvironnements, des systèmes agropastoraux et évolution des territoires ruraux, Thèse de Doctorat, Université de Franche-Comté, France.

Munaut, A.-V. 1967. Recherches paléo-écologiques en basse et moyenne Belgique. Acta Geographica Lovaniensia 6, 191 p.

Oberdorfer, E., 1994. Pflanzensoziologische Exkursionsflora, 7 , Auflage, Ulmer, UTB für Wissenschaft.

Pasternak, R., 1991. Hafer aus dem mittelalterlichen Schleswig, Offa, 48, p. 363-380.

Périgord, M., 1994. Essai de traitement géographique des paysages : l'exemple du Limousin, Norois, 41, (161), p. 235-256.

Petit, F., Fredon, J.-J., 2001. Rapport d'anthracologie archéologique sur le site gallo-romain des Sagnes à Pontarion (Creuse). In G. Lintz (dir.), La nécropole gallo-romaine des Sagnes à Pontarion (Creuse), éd. Association des Publications Chauvinoises, Mémoires 20, p. 197-203.

Riera i Mora, S., 1994. Evolció del paisatge vegetal holocè al pla de Barcelona, a partir de les dades pol. liniques, Departament de Prehistòria, Història Antiga i Arqueologia, Facultat de Geografia i Història, Thèse de Doctorat, Univeritat de Barcelona, Espagne.

Roger, J., 1995. La nécropole gallo-romaine de la Bétoulle (SaintMaurice-La-Souterraine), Aquitania, 13, p. 155-178.

Rösch, M., 1997. Ackerbau und Ernährung. Pflanzenreste aus alamannischen Siedlungen, In : Die Alamannen, hrsg. Archäologisches Landesmuseum Baden-Württemberg, Stuttgart, p. 323-330.

Rösch, M., 1998. Naturhistorischer Quellenwert des Bodens, in I. Kowarik, E. Schmidt, B. Sigel (dir.), Naturschutz und Denkmalpflege. Wege zu einem Dialog im Garten, 18, p. 309318.

Rösch, M., 1999. Archäobotanische Belege für frühmittelalterlichen Gartenbau in Südwestdeutschland, in R. Rolle, F. M. Andraschko (dir.), Frühe Nutzung pflanzlicher Ressourcen, Internationales Symposium Duderstadt (1994), p. 61-69 et 159.

Rösch, M., 2005. Landwirtschaft und Kulturlandschaft im Enztal während acht Jahrtausendenn, in Stadtarchiv Mühlacker (Hrsg.), Historische Streiflichter. Teil 2, von der urzeitlichen Landwirtschaft über die Gastwirtschaften zum Stadtjubiläum (Heidelberg/Ubstadt-Weiher/Basel 2005), p. 69-93.
RösCH, M., 2006a. Eisenzeitliche Pflanzenreste aus dem keltischen Oppidum Heidengraben bei Grabenstetten, Kreis Reutlingen, in T. Knopf (dir.), Der Heidengraben bei Grabenstetten. Archäologische Untersuchungen zur Besiedlungsgeschichte, Verlag Dr. Rudolf Habelt GmbH, Bonn, p. 231-261.

Rösch, M., 2006b. Die Gärten der Alamannen. Bodenfunde zeigen ein neues Bild vom Pflanzenbau nördlich der Alpen, Denkmalpflege in Baden-Württemberg, 3, p. 166-171.

Rösch, M., Fischer, E., Müller, H., Sillmann, M., Stika, H.P., 2008. Botanische Untersuchungen zur eisenzeitlichen Landnutzung im südlichen Mitteleuropa, Forschungen und Berichte zur Vor-und Frühgeschichte in Baden-Württemberg 101, (Festschrift Jörg Biel), p. 319-347.

RuAs, M.-P., 1992a. The archaeobotanical record of cultivated and collected plants of economic importance from medieval sites in France, in J.-P. Pals, J. Buurman, M. Van Der Veen, Festschrift for Professor Van Zeist, Elsevier Publishers Sciences. Review of Palaeobotany and Palynology. An International Journal, Amsterdam, No spécial, 73 (1-4), p. 301-314.

RuAs, M.-P., 1992b. Les plantes exploitées en France au Moyen Âge d'après les semences archéologiques, Plantes et cultures nouvelles en Europe occidentale, au Moyen Âge et à l'époque moderne Flaran, $12^{\mathrm{e}}$ Journées Internationales d'Histoire de l'abbaye de Flaran (Gers), septembre 1990, éd. Comité Départemental du Tourisme du Gers, Auch. Flaran, 12, p. 9-35.

RuAS, M.-P., 1995. Étude de paléoenvironnement sur le presbytère. Légumes, épices et fruits consommés au presbytère de Rigny dans la première moitié du $\mathrm{XvI}^{\mathrm{e}} \mathrm{s}$. : les données carpologiques, In E. Zadora-Rio, H. Galinié (dir.), La fouille de l'ancien centre paroissial de Rigny (commune de Rigny-Ussé, Indre-et-Loire) - Deuxième rapport préliminaire (1992-1994). Revue Archéologique du Centre de la France, 34, p. 229-246.

Ruas, M.-P., 1998. Les plantes consommées au Moyen Âge en France méridionale d'après les semences archéologiques, In $\mathrm{S}$. Campech et N. Pousthoumis-Dalle (éds), Usages et gồts culinaires au Moyen Âge en Languedoc et en Aquitaine, Actes du colloque (Carcassonne, 1996), Archéologie du Midi médiéval, 15-16, p. 179-204.

Ruas, M.-P., 2000. Cultures, importations et alimentation d'après les semences, In $\mathrm{G}$. Berthaud, Mazières-en-Mauges gallo-romain (Maine-et-Loire). Un quartier à vocation artisanale et domestique. Éd. ARDA, Angers, p. 199-226.

RuAs, M.-P., 2001. Note sur les semences du dolmen de la Lue à Berneuil (Haute-Vienne), Bulletin Préhistoire du Sud-Ouest, 8, p. 193-194.

RuAS, M.-P., 2005. Aspects of early medieval farming from sites in Mediterranean France, Vegetation History and Archaeobotany, 14 , p. 400-415.

Ruas, M.-P., Bouby, L., Pradat, B., 2005-2006. Les restes de fruits dans les dépôts archéologiques, in Thème 3 : Les fruits

ArcheoSciences, revue d'archéométrie, 35, 2011, p. 65-92 
sur la table, Dossier spécial : la fruticulture, Archéologie du Midi Médiéval, 23-24, p. 145-193.

Ruas, M.-P., Marinval, P., 1991. Alimentation végétale et agriculture d'après les semences archéologiques (de 9000 ans avant J.-C. au Xv $\mathrm{xv}^{\mathrm{e}}$ siècle), in J. Guilaine (dir.), Pour une archéologie agraire, Éditions Armand Colin, Paris, p. 409-439.

Ruas, M.-P., Pradat, B., 2001. Les semences découvertes : plantes attestées et origines des déchets, In : Cattedu I. (dir.), Les habitats carolingiens de Montours et la Chapelle Saint-Aubert (Ille-et-Vilaine), éd. Documents d'Archéologie Française, série Archéologie Préventive, Paris, 89, p. 65-79 et 219-221.

Stebich, M., Brüchmann, C., Kulbe, T., Negendank, J. F. W., 2005. Vegetation history, human impact and climatic change during the last 700 years recorded in annually laminated sediments of Lac Pavin, France. Review of Palaeobotany and Palynology, 133, p. 115-133.

Tardiveau, D., Vuaillat, D., Evin, J., Richard, H., 1990. Recherches archéologiques dans les Monts de Blond (HauteVienne). Apports chronologiques. Notes préliminaires. 1. L'abri des Fées (Cieux, Haute-Vienne). Revue Archéologique du Centre de la France, 29, (2), p. 173-175.

Tits-Dieuaide, M.-J., 1994. Les campagnes d'Europe occidentale au Moyen Âge,. In E. Le Roy Ladurie (dir.), Paysages, paysans. L'art et la terre en Europe du Moyen Âge au XX' siècle, éd. Bibliothèque Nationale de France, Réunion des Musées Nationaux, p. 27-35.

Toledo i Mur, A., Milor, F., Roger, J., Petit, F., Bet, P., Witmann, A., Cabanis, M., avec la collaboration de Vigneron, M. et Miailhe, V., 2004. Un site cultuel et funéraire de la protohistoire aux premiers temps de la période gallo-romaine : les Châtaigneraies, Saint-Germain-les-Vergnes (Corrèze), Travaux d'Archéologie Limousine, 24, p. 7-51.

Toupet, C. et Lemaitre, P. avec la collaboration de Costa, L., Krier, V., Limondin, N., Gebhart, A., Barbet, V., Leroyer, C., Allenet, G., Yeny, E., Vangele, F., Michelland, M., Manceau, C., Marquis, P., 2003. Vignobles et modes d'exploitation viticoles antiques dans le Nord de la Gaule. L'exemple de Bruyères-sur-Oise (Val d'Oise) : une relecture, Revue Archéologique de Picardie, 1/2, p. 209-223.

Valadas, B., 1982. L'approche des paléoenvironnements du Limousin : intérêt et apports de deux méthodes, Travaux d'Archéologie Limousine, 3, p. 53-57.

Valadas, B., Marambat, L., 1999. Aux origines du paysage en Montagne limousine : les tourbières des Meillauds (SaintYrieix-la-Montagne, Creuse), et de Combe-l'Epine (Royèrede-Vassivière, Creuse), Travaux d'Archéologie Limousine, 19, p. 69-76.

Van Der Veen, M., 2005. Gardens and fields : the intensity and scale of food production, World Archaeology, 37, (2), p. 157163.

ArcheoSciences, revue d'archéométrie, 35, 2011, p. 65-92
VAN ZeIST, W., 1991. Economic aspects, in W. van Zeist, K. Wasilykowa, K.-E. Behre (dir.), Progress in Old World Paleoethnobotany. An retrospective view on the occasion of 20 years of the International Work Group for Palaeoethnobotany, A.A. Balkema, Rotterdam, Brookfield, p. 109-130.

Van Zeist W., De Roller, G. J., Palfenier-Vegter, R. M., Harsema, O. H., During, H., 1986. Plant remains from medieval sites in Drenthe, The Netherlands, Helinium, 26, p. 226-274.

Van Zeist, W., Palfenier-Vegter, R. M., 1979. Agriculture in medieval Gasselte, Palaeohistoria, 21, p. 267-299.

Vanderpooten, M., 2002. La place des légumineuses parmi les cultures et dans l'alimentation animale et humaine, de l'Antiquité au XIx ${ }^{\mathrm{e}}$ siècle, Autour d'Olivier de Serres. Pratiques agricoles et pensée agronomique, du Néolithique aux enjeux actuels. Actes du colloque international, Pradel 2002, éd. Association d'Histoire des Sociétés Rurales, p. 173-190.

Visset, L., Cyprien, A.-L., Ouguerram, A., Barbier, D., Bernard, J., 2004. Les indices polliniques d'anthropisation précoce dans l'Ouest de la France. Le cas de Cerealia, Fagopyrum et Juglans, In H. Richard (dir.), Néolithisation précoce. Premières traces d'anthropisation du couvert végétal à partir des données polliniques, Besançon, PUFC, p. 69-79.

Visset, L., Cyprien, A.-L., Carcaud, N., Bernard, J., Ouguerram, A., 2005. Paysage végétal dans le Bassin de la Loire moyenne du Tardiglaciaire à l'Actuel, Le Journal de Botanique, ed. Société Botanique de France, 29, p. 41-51.

Walter, H., Lieth, H., Rehder, H., 1960. KlimadiagrammWeltatlas, Gustav Fischer Verlag, Jena.

Wiethold, J., 1998. Recherches archéobotaniques en France du Centre-Est, Rapport annuel d'activité scientifique 1998 du Centre archéologique du Mont Beuvray, Glux-en-Glenne, Centre archéologique du Mont Beuvray, décembre 1998, p. 217-240.

Wiethold, J., 1999. Recherches archéobotaniques, Rapport annuel d'activité scientifique 1999 du Centre archéologique du Mont Beuvray, Glux-en-Glenne, Centre archéologique du Mont Beuvray, décembre 1999, p. 243-257.

Zech-Matterne V., Bouby L., Bouchette A., Cabanis M., Derreumaux M., Durand F., Marinval P., Pradat B., Sellami M.-F., Wiethold J., 2009. L'agriculture du $\mathrm{vi}^{\mathrm{e}}$ au $\mathrm{I}^{\mathrm{er}}$ siècle avant J.-C. en France : Etat des recherches carpologiques sur les établissements ruraux, in Actes du XXXIe Colloque International de l'Association Française pour l'Étude de l'âge du Fer, 17-20 mai 2007, Chauvigny (Vienne), p. 383-416. 